\title{
A Comparison Of Stochastic Systems With Different Types Of Delays
}

\author{
H.T. Banks, Jared Catenacci and Shuhua Hu \\ Center for Research in Scientific Computation, \\ North Carolina State University \\ Raleigh, NC 27695-8212
}

March 26, 2013

\begin{abstract}
In this paper we investigate the effects of different types of delays, a fixed delay and a random delay, on the dynamics of stochastic systems as well as their relationship with each other in the context of a just-in-time network model. The specific example on which we focus is a pork production network model. We numerically explore the corresponding deterministic approximations for the stochastic systems with these two different types of delays. Numerical results reveal that the agreement of stochastic systems with fixed and random delays depend upon the population size and the variance of the random delay, even when the mean value of the random delay is chosen the same as the value of the fixed delay. When the variance of the random delay is sufficiently small, the histograms of state solutions to the stochastic system with a random delay are similar to those of the stochastic model with a fixed delay regardless of the population size. We also compared the stochastic system with a Gamma distributed random delay to the stochastic system constructed based on the Kurtz's limit theorem from a system of deterministic delay differential equations with a Gamma distributed delay. We found that with the same population size the histogram plots for the solution to the second system appear more dispersed than the corresponding ones obtained for the first case. In addition, we found that there is more agreement between the histograms of these two stochastic systems as the variance of the Gamma distributed random delay decreases.
\end{abstract}

Key Words: systems with delays, Markov Chain stochastic vs. deterministic approximations, Kurtz limit theorem, fixed vs. random delays, Gamma distribution and the linear chain trick.

\section{Introduction}

Continuous time Markov Chain models are widely used to model physical and biological processes (e.g., see $[1,3]$ ). These models are typically (and most appropriately) used when dealing with dynamic systems involving low species count. In stochastic modeling one often wishes to know whether or not the stochastic system can be approximated by a deterministic one when the population size is sufficiently large. Theory established by Kurtz (e.g., $[14,15,16]$ ), provides a way to construct a deterministic system to approximate density dependent continuous time Markov Chains as the population size grows large (this result is often called the Kurtz's limit theorem, see details below). In general, deterministic systems are much easier to analyze compared to stochastic systems. Techniques, such as parameter estimation methods, are well developed for deterministic systems, whereas parameter estimation is much more difficult in a stochastic framework (e.g., see [22]).

Delays occur and are important in many physical and biological processes. For example, recent studies show that delayed-induced stochastic oscillations can occur in certain elements of gene regulation networks [10]. These are similar to delay-induced phenomena recognized for many years in mechanical systems (e.g., see $[19,20,21])$. More recent applications have focused on concerns that delays might be of practical importance in general supply networks in investigations of a wide range of perturbations (either accidental or deliberate). Examples include a node being rendered inoperable due to bad weather, and technical difficulties in communication systems. Hence, continuous time Markov chain models with delays incorporated (simply 
referred to stochastic models with delays in this paper) have enjoyed considerable research attention in the past decade, especially the efforts on the development of algorithms to simulate such systems (e.g., $[2,7,10,24])$. However, to the best of our knowledge, it appears that there is a dearth of efforts on the convergence of solutions to stochastic system with delays as the sample size goes to infinity (that is, an analogy of Kurtz's limit theorem). The two works that we found are the results presented in [9] and [26]. Specifically, Bortolussi and Hillston [9] extended the Kurtz's limit theorem to the case where fixed delays are incorporated into a density dependent continuous time Markov chain. Schlicht and Winkler [26] showed that if all the transition rates are linear, then the mean solution of stochastic system with random delays can be described by deterministic differential equations with distributed delays. However, to our knowledge, there are still no theoretical results on the convergence of solutions to a general stochastic system with random delays. That is, there is still no analog of the Kurtz's limit theorem for a general stochastic system with random delays.

As a first step, in this paper we numerically explore the corresponding deterministic differential equations for the stochastic systems with delays, and investigate the effects of different types of delays on the dynamics of stochastic systems as well as their relationship with each other. Specifically, we do this in the context of an extension of the pork production network model in [4] to incorporate delays which account for the phenomenon that the movement from one node to the next is often not instantaneous nor deterministic. Before addressing our main tasks, we shall use this introduction to give a brief review of Kurtz's limit theorem in Section 1.1 and the original pork production model in Section 1.2.

\subsection{The Kurtz's limit theorem}

Let $\nu$ be a positive integer number, and $\mathbb{Z}^{\nu}$ be the set of $\nu$-dimensional column vectos with integer components. Suppose for each positive number $N,\left\{\mathbf{X}^{N}(t), t \geq 0\right\}$ with state space $\mathcal{X}^{N} \subset \mathbb{Z}^{\nu}$ is a continuous time Markov chain with transition rate $\lambda_{j}\left(\mathbf{x}^{N}\right)$ at the $j$ th transition (often referred to as reactions in the biochemistry literature), $j=1,2,3, \ldots, M$ with $M$ being the number of transitions. In other words, for any small time interval $\Delta t$ we have

$$
\operatorname{Prob}\left\{\mathbf{X}^{N}(t+\Delta t)=\mathbf{x}^{N}+\mathbf{v}_{j} \mid \mathbf{X}^{N}(t)=\mathbf{x}^{N}\right\}=\lambda_{j}\left(\mathbf{x}^{N}\right) \Delta t+o(\Delta t), \quad j=1,2, \ldots, M,
$$

where $\mathbf{v}_{j}=\left(v_{1 j}, v_{2 j}, v_{3 j}, \ldots, v_{\nu j}\right)^{T} \in \mathbb{Z}^{\nu}$ with $v_{i j}$ denoting the change in state variable $X_{i}^{N}$ caused by the $j$ th transition. This family of continuous time Markov chain is called density dependent if and only if there exist continuous functions $f_{j}: \mathbb{R}^{\nu} \rightarrow \mathbb{R}$ such that

$$
\lambda_{j}(\mathbf{x})=N f_{j}(\mathbf{x} / N), \quad j=1,2, \ldots, M .
$$

This process can be characterized by standard Poisson processes (e.g., see $[3,12])$, that is, $\mathbf{X}^{N}(t)$ can be written as

$$
\mathbf{X}^{N}(t)=\mathbf{X}^{N}(0)+\sum_{j=1}^{M} \mathbf{v}_{j} Y_{j}\left(\int_{0}^{t} \lambda_{j}\left(\mathbf{X}^{N}(s)\right) d s\right),
$$

where $\left\{Y_{j}(t), t \geq 0\right\}, j=1,2, \ldots, M$ are independent standard Poisson processes. Let $\mathbf{C}^{N}(t)=\mathbf{X}^{N}(t) / N$. Then we obtain another continuous time Markov chain $\left\{\mathbf{C}^{N}(t), t \geq 0\right\}$. Define

$$
\mathbf{g}(\mathbf{c})=\sum_{j=1}^{M} \mathbf{v}_{j} f_{j}(\mathbf{c})
$$

Then by (2) and (3), one can use the strong law of large numbers to show that with some mild conditions on $\mathrm{g}$ and $f_{j}$ the processes $\left\{\mathbf{C}^{N}(t), t \geq 0\right\}$ converges to a deterministic process that is the solution of the system of ordinary differential equations

$$
\dot{\mathbf{c}}(t)=\mathbf{g}(\mathbf{c}), \quad \mathbf{c}(0)=\mathbf{c}_{0} .
$$

This result was originally shown by Kurtz $[14,15]$, and hence it is often referred to as the Kurtz's Limit Theorem. For the convenience of the readers, we will state this result in the following theorem (e.g., see $[3,17]$ for details). 
Theorem 1. Suppose that $\lim _{N \rightarrow \infty} \mathbf{C}^{N}(0)=\mathbf{c}_{0}$ and for any compact set $\Omega \subset \mathbb{R}^{\nu}$ there exists a positive constant $\eta_{\Omega}$ such that

Then we have

$$
|\mathbf{g}(\mathbf{c})-\mathbf{g}(\tilde{\mathbf{c}})| \leq \eta_{\Omega}|\mathbf{c}-\tilde{\mathbf{c}}|, \quad \mathbf{c}, \tilde{\mathbf{c}} \in \Omega
$$

$$
\lim _{N \rightarrow \infty} \sup _{t \leq t_{f}}\left|\mathbf{C}^{N}(t)-\mathbf{c}(t)\right|=0 \text { a.s. for all } t_{f}>0,
$$

where $\mathbf{c}(t)$ denotes the unique solution to (4).

Theorem 1 indicates that the convergence is in the sense of convergence almost surely. It is worth noting that in Kurtz's original work $[14,15]$ the convergence is in the sense of convergence in probability. In addition, it should be noted that based on the problem considered, the parameter $N$ can be interpreted as the total number in a population, the volume of the population occupied, or some other scaling factor. Hence, the parameter $N$ is often called the population size, the sample size, or scaling parameter. For notational convenience, we suppress the dependence on $N$ in much of the remainder of this paper (i.e., if no confusion occurs). Thus, we simply denote $\mathbf{X}^{N}$ by $\mathbf{X}$ and $\mathbf{C}^{N}$ by $\mathbf{C}$.

\subsection{The original pork production network model}

In current production methods for livestock based on a just-in-time philosophy, animals are grown in different areas, and are moved from one farm to another depending on their age. Unfortunately, shocks propagate rapidly through such systems, and may cause devastating effects on their performance. For example, stopping movement of animals to and from a farm with animals infected by a disease will have effects that quickly spread through the whole system: nurseries supplying the farm will have nowhere to send their animals as they grow up, and finishers and slaughterhouses supplied by the farm will have their supply interrupted. Hence, it is of great interest to identify bottlenecks in the production and feed supply chain, and to test potential mitigation tools, procedures, and practices to increase the resilience of animal agriculture to catastrophic events. Motivated by this, a stochastic pork production network model was developed in [4] to investigate how small perturbations to the agricultural supply system would affect its overall performance.

We give a brief review of the the pork production model developed in [4]; interested readers can refer to [4] for more information. In this model, four nodes of production are considered: sows, nurseries, finishers, and slaughterhouses. The movement of pigs from one node to the next is assumed to occur only in the forward direction. That is, from sows to nurseries, from nurseries to finishers and from finishers to slaughterhouses. The population $(N)$ is assumed to remain constant, and thus, the number of deaths that occur at the slaughterhouses is taken to be the same as number of births at the sows node. The evolution of the system is modeled using a continuous time Markov Chain with states at time $t$ denoted by $\mathbf{X}(t)=\left(X_{1}(t), X_{2}(t), X_{3}(t), X_{4}(t)\right)^{T}$, where $X_{i}(t)$ is the number of pigs at the $i$ th node at time $t$.

Furthermore, the model assumes that there is a capacity constraint $\left(L_{i}\right)$ at each node with a maximal exit constraint $S_{m}$ at node 4 . Let $\mathbf{e}_{i} \in \mathbb{Z}^{4}$ be the $i$ th unit column vector, that is, the $i$ th entry of $\mathbf{e}_{i}$ is 1 and all the other entries are zeros, $i=1,2,3,4$. The transition rate $\lambda_{j}(\mathbf{x})$ and the corresponding state change vector $\mathbf{v}_{j}$ at the $j$ th transition, $j=1,2,3,4$, are given by

$$
\begin{aligned}
& \lambda_{1}(\mathbf{x})=k_{1} x_{1}\left(L_{2}-x_{2}\right)_{+}, \quad \mathbf{v}_{1}=-\mathbf{e}_{1}+\mathbf{e}_{2}, \\
& \lambda_{2}(\mathbf{x})=k_{2} x_{2}\left(L_{3}-x_{3}\right)_{+}, \quad \mathbf{v}_{2}=-\mathbf{e}_{2}+\mathbf{e}_{3}, \\
& \lambda_{3}(\mathbf{x})=k_{3} x_{3}\left(L_{4}-x_{4}\right)_{+}, \quad \mathbf{v}_{3}=-\mathbf{e}_{3}+\mathbf{e}_{4}, \\
& \lambda_{4}(\mathbf{x})=k_{4} \min \left(x_{4}, S_{m}\right), \quad \mathbf{v}_{4}=-\mathbf{e}_{4}+\mathbf{e}_{1},
\end{aligned}
$$

where $(z)_{+}=\max (z, 0)$, and $k_{i}$ is the service rate at node $i, i=1,2,3,4$. Then by (3) we know that the pork production network can be described by the following stochastic system.

$$
\begin{aligned}
& X_{1}(t)=X_{1}(0)-Y_{1}\left(\int_{0}^{t} \lambda_{1}(\mathbf{X}(s)) d s\right)+Y_{4}\left(\int_{0}^{t} \lambda_{4}(\mathbf{X}(s)) d s\right) \\
& X_{2}(t)=X_{2}(0)-Y_{2}\left(\int_{0}^{t} \lambda_{2}(\mathbf{X}(s)) d s\right)+Y_{1}\left(\int_{0}^{t} \lambda_{1}(\mathbf{X}(s)) d s\right) \\
& X_{3}(t)=X_{3}(0)-Y_{3}\left(\int_{0}^{t} \lambda_{3}(\mathbf{X}(s)) d s\right)+Y_{2}\left(\int_{0}^{t} \lambda_{2}(\mathbf{X}(s)) d s\right) \\
& X_{4}(t)=X_{4}(0)-Y_{4}\left(\int_{0}^{t} \lambda_{4}(\mathbf{X}(s)) d s\right)+Y_{3}\left(\int_{0}^{t} \lambda_{3}(\mathbf{X}(s)) d s\right) .
\end{aligned}
$$


There are a number of algorithms that can be used to simulate a stochastic system such as (7) (e.g., see [23] for a recent review of such algorithms). Here we will outline one of them, the modified next reaction method (NRM) algorithm, which is a modification to the next reaction method of Gibson and Bruck [11]. The NRM algorithm was developed by Anderson in [2], wherein the next reaction method of Gibson and Bruck was modified to make more explicit use of the "internal times", which are defined as $\int_{0}^{t} \lambda_{i}(\mathbf{X}(s)) d s$ for each transition. One of the advantages of the modified next reaction algorithm is that it can be easily extended to simulate stochastic systems with time dependent transition rates whereas the next reaction method can not. In addition, the NRM algorithm can be altered to simulate stochastic systems with fixed delays, and further altered to simulate stochastic systems with random delays.

Suppose the stochastic system to be simulated has $M \geq 1$ transitions with transition rates $\lambda_{i}(\mathbf{x})$ for $i=1,2, \ldots, M$. Then the NRM algorithm is given as follows.

\section{Algorithm 1: Modified Next Reaction Method}

1. Set the initial condition for each state at $t=0$. Set $T_{i}=0$ for $i=1,2, \ldots, M$.

2. Calculate each transition rate $\lambda_{i}$ at the given state for $i=1,2, \ldots, M$.

3. Generate $M$ independent, uniform $(0,1)$ random numbers $r_{i}$.

4. Set $P_{i}=\ln \left(1 / r_{i}\right)$ for $i=1,2, \ldots, M$.

5. Set $\Delta t_{i}=\left(P_{i}-T_{i}\right) / \lambda_{i}$ for $i=1,2, \ldots, M$.

6. Set $\Delta=\min _{1 \leq i \leq M}\left(\Delta t_{i}\right)$, and let $l$ be the reaction which obtains the minimum.

7. Set $t=t+\Delta$.

8. Update the system based on the reaction $l$.

9. Set $T_{i}=T_{i}+\lambda_{i} \Delta$ for $i=1,2, \ldots, M$.

10. For transition $l$, choose a uniform random number $(0,1), r$, and set $P_{l}=P_{l}+\ln (1 / r)$.

11. Recalculate each transition rate $\lambda_{i}$ at the given new state for $i=1,2, \ldots, M$.

12. Return to step 5.

As in [4], if we rescale the stochastic system (7) in such a way that instead of following the number of pigs at each node we track the concentration of pigs (i.e., $\mathbf{C}(t)=\mathbf{X}(t) / N$ where $N=\Sigma_{i=1}^{4} X_{i}(t)$ ), then by Kurtz's limit theorem we know that the appropriate rescaling allows us to obtain an approximating system of ordinary differential equations (ODE's) for the scaled stochastic system $\mathbf{C}(t)$. Rescale the constants as follows:

$$
\begin{aligned}
& \kappa_{4}=k_{4}, \quad \kappa_{i}=N k_{i}, \quad i=1,2,3 \\
& s_{m}=S_{M} / N, \quad l_{i}=L_{i} / N .
\end{aligned}
$$

Then the resulting approximating system of ODE's is given by

$$
\begin{aligned}
& \dot{c}_{1}(t)=-\kappa_{1} c_{1}(t)\left(l_{2}-c_{2}(t)\right)_{+}+\kappa_{4} \min \left(c_{4}(t), s_{m}\right) \\
& \dot{c}_{2}(t)=-\kappa_{2} c_{2}(t)\left(l_{3}-c_{3}(t)\right)_{+}+\kappa_{1} c_{1}(t)\left(l_{2}-c_{2}(t)\right)_{+} \\
& \dot{c}_{3}(t)=-\kappa_{3} c_{3}(t)\left(l_{4}-c_{4}(t)\right)_{+}+\kappa_{2} c_{2}(t)\left(l_{3}-c_{3}(t)\right)_{+} \\
& \dot{c}_{4}(t)=-\kappa_{4} \min \left(c_{4}(t), s_{m}\right)+\kappa_{3} c_{3}(t)\left(l_{4}-c_{4}(t)\right)_{+} \\
& \mathbf{c}(0)=\mathbf{c}_{0},
\end{aligned}
$$

where $\mathbf{c}(t)=\left(c_{1}(t), c_{2}(t), c_{3}(t), c_{4}(t)\right)^{T}$, and $\mathbf{c}_{0}=\left(c_{10}, c_{20}, c_{30}, c_{40}\right)^{T}$.

\section{The Pork Production Network Model With A Fixed Delay}

We see from Section 1.2 that the assumption made on the original pork production network model [4] is that the transition from one node to the next is made instantaneously. It was made clear in [4] that this is a simplifying assumption, and that incorporating delays would give a more realistic model due to the possible 
long distance between the nodes, bad weather or some other disruptions/interruptions. Presented here is a first attempt to account for delays in the following way. Assume that all transitions occur instantaneously except for the arrival of pigs transitioning from node 1 to 2 . That is, the pigs leave node 1 immediately, but the time of arrival at node 2 is delayed. For simplicity, we only consider a delay in one of the transitions, but depending on the physical proximity of the nodes, it may be a reasonable assumption to have delays in even more of the transitions.

\subsection{The stochastic model with a fixed delay}

As a first and simplest consideration, we take the delayed time of arrival at node $2, \tau$, to be a fixed value. If we assume that the process starts from $t=0$ (that is, $\mathbf{X}(t)=0$ for $t<0$ ), then this results in a stochastic model with a fixed delay given by

$$
\begin{aligned}
& X_{1}(t)=X_{1}(0)-Y_{1}\left(\int_{0}^{t} \lambda_{1}(\mathbf{X}(s)) d s\right)+Y_{4}\left(\int_{0}^{t} \lambda_{4}(\mathbf{X}(s)) d s\right) \\
& X_{2}(t)=X_{2}(0)-Y_{2}\left(\int_{0}^{t} \lambda_{2}(\mathbf{X}(s)) d s\right)+Y_{1}\left(\int_{0}^{t} \lambda_{1}(\mathbf{X}(s-\tau)) d s\right) \\
& X_{3}(t)=X_{3}(0)-Y_{3}\left(\int_{0}^{t} \lambda_{3}(\mathbf{X}(s)) d s\right)+Y_{2}\left(\int_{0}^{t} \lambda_{2}(\mathbf{X}(s)) d s\right) \\
& X_{4}(t)=X_{4}(0)-Y_{4}\left(\int_{0}^{t} \lambda_{4}(\mathbf{X}(s)) d s\right)+Y_{3}\left(\int_{0}^{t} \lambda_{3}(\mathbf{X}(s)) d s\right) .
\end{aligned}
$$

Note that $\lambda_{1}$ only depends on the state. Hence, the assumption of $\mathbf{X}(t)=0$ for $t<0$ leads to $\lambda_{1}(\mathbf{X}(t))=0$ for $t<0$. The interpretation of this stochastic model with a fixed delay (9) is as follows. When any of the transitions $\lambda_{2}, \lambda_{3}$ or $\lambda_{4}$ fires at time $t$, the system is updated accordingly at time $t$. When the transition $\lambda_{1}$ fires at time $t$, one unit is subtracted from the first node. Since the completion of the transition is delayed, at time $t+\tau$ the unit is added onto node 2 .

\subsection{Algorithms for stochastic systems with fixed delays}

In this section we outline one possible algorithm that may be used to simulate a stochastic system such as (9) with fixed delays. This modified next reaction method for systems with delays algorithm is given in [2]. In general, there are three types of reactions to consider. First are the reactions with no delay (ND). Second are the reactions where they only affect the state of the system upon completion (CD). And finally, the reactions where the state of the system is affected at the initiation and the completion of the reaction (ICD). In the example case here the delayed reaction is an ICD. As before let $M \geq 1$ be the number of transitions with transition rates $\lambda_{i}(\mathbf{x})$ for $i=1,2, \ldots, M$. In addition, if the $j$ th transition is a delayed one, then we let $\tau_{j}$ denote the delay time between the initiation and completion for this transition.

\section{Algorithm 2: Modified Next Reaction Method For Stochastic Systems With Fixed Delays}

1. Set the initial condition for each state at $t=0$. Set $T_{i}=0$, and the array $\mathbf{m}_{i}=(\infty)$ for $i=1,2, \ldots, M$.

2. Calculate each transition rate $\lambda_{i}$ at the given state for $i=1,2, \ldots, M$.

3. Generate $M$ independent, uniform $(0,1)$ random numbers $r_{i}$.

4. Set $P_{i}=\ln \left(1 / r_{i}\right)$ for $i=1,2 \ldots, M$.

5. Set $\Delta t_{i}=\left(P_{i}-T_{i}\right) / \lambda_{i}$ for $i=1,2, \ldots, M$.

6. Set $\Delta=\min _{1 \leq i \leq M}\left(\Delta t_{i}, \mathbf{m}_{i}(1)-t\right)$.

7. Set $t=t+\Delta$.

8. If $\Delta t_{l}$ obtained the minimum in step 6 then do the following. If the $l$ reaction is a ND, then update the system according to the reaction $l$. If reaction $l$ is a CD then store the time $t+\tau_{l}$ in the second to last position in $\mathbf{m}_{l}$. If reaction $l$ is a ICD then update the system according to the initiation of the $l$ reaction and store $t+\tau_{l}$ in the second to last position in $\mathbf{m}_{l}$.

9. If $\mathbf{m}_{l}(1)-t$ obtained the minimum in step 6 then update the system according the completion of reaction $l$ and delete the first element in $\mathbf{m}_{l}$. 
10. Set $T_{i}=T_{i}+\lambda_{i} \Delta$ for $i=1,2, \ldots, M$.

11. For transition $l$ which initiated, choose a uniform random number $(0,1), r$, and set $P_{l}=P_{l}+\ln (1 / r)$.

12. Recalculate each transition rate $\lambda_{i}$ at the given new state for $i=1,2, \ldots, M$.

13. Return to step 5.

\subsection{The corresponding deterministic system for the stochastic model with a fixed delay}

In [9], Bortolussi and Hillston extended the Kurtz's limit theorem to a scenario with fixed delays incorporated into a density dependent continuous time Markov chain, where the convergence is in the sense of convergence in probability. We will use our pork production model (9) to illustrate this theorem (referred to as the BH limit theorem). Using the same rescaling procedure as before $(\mathbf{C}(t)=\mathbf{X}(t) / N$ with $\mathbf{X}(t)$ described by (9)), an approximating deterministic system can be constructed based on the BH limit theorem for the scaled stochastic system with a fixed delay. This approximating deterministic system is given by

$$
\begin{aligned}
& \dot{c}_{1}(t)=-\kappa_{1} c_{1}(t)\left(l_{2}-c_{2}(t)\right)_{+}+\kappa_{4} \min \left(c_{4}(t), s_{m}\right) \\
& \dot{c}_{2}(t)=-\kappa_{2} c_{2}(t)\left(l_{3}-c_{3}(t)\right)_{+}+\kappa_{1} c_{1}(t-\tau)\left(l_{2}-c_{2}(t-\tau)\right)_{+} \\
& \dot{c}_{3}(t)=-\kappa_{3} c_{3}(t)\left(l_{4}-c_{4}(t)\right)_{+}+\kappa_{2} c_{2}(t)\left(l_{3}-c_{3}(t)\right)_{+} \\
& \dot{c}_{4}(t)=-\kappa_{4} \min \left(c_{4}(t), s_{m}\right)+\kappa_{3} c_{3}(t)\left(l_{4}-c_{4}(t)\right)_{+} .
\end{aligned}
$$

We note that this approximating deterministic system is no longer a system of ordinary differential equations, but rather a system of delay (ordinary) differential equations with a fixed delay. The delay differential equation is a direct result of the delay term present in (9). Since there is a delay term, the system is dependent on the previous states, for this reason it is necessary to have some past history functions as initial conditions. It should be noted that past history functions should not be chosen in an arbitrary fashion as they should capture the limit dynamics of the scaled stochastic system with a fixed delay.

Next we illustrate how to construct the initial conditions for the delay differential equation (10). We observe that in the interval $[0, \tau]$ the delay term has no affect, thus we can ignore the delay term in this interval. This yields a stochastic system with no delays, the concentration of which can be approximated by a system of ODE's as was done previously. This gives the deterministic system

$$
\begin{aligned}
& \dot{c}_{1}(t)=-\kappa_{1} c_{1}(t)\left(l_{2}-c_{2}(t)\right)_{+}+\kappa_{4} \min \left(c_{4}(t), s_{m}\right) \\
& \dot{c}_{2}(t)=-\kappa_{2} c_{2}(t)\left(l_{3}-c_{3}(t)\right)_{+} \\
& \dot{c}_{3}(t)=-\kappa_{3} c_{3}(t)\left(l_{4}-c_{4}(t)\right)_{+}+\kappa_{2} c_{2}(t)\left(l_{3}-c_{3}(t)\right)_{+} \\
& \dot{c}_{4}(t)=-\kappa_{4} \min \left(c_{4}(t), s_{m}\right)+\kappa_{3} c_{3}(t)\left(l_{4}-c_{4}(t)\right)_{+} \\
& \mathbf{c}(0)=\mathbf{c}_{0}
\end{aligned}
$$

for $t \in[0, \tau]$. We let $\mathbf{\Phi}(t)$ denote the solution to (11) and thus we have that $\mathbf{C}(t)$ converges to $\boldsymbol{\Phi}(t)$ as $N \rightarrow \infty$ on the interval $[0, \tau]$.

In the interval $\left[\tau, t_{f}\right]$, where $t_{f}$ is the final time, the delay has an affect, so we approximate with the DDE system (10), and the solution $\boldsymbol{\Phi}(t)$ to the ODE (11) on the interval $[0, \tau]$ serves as the initial function. Explicitly the system can be written as

$$
\begin{aligned}
\dot{c}_{1}(t) & =-\kappa_{1} c_{1}(t)\left(l_{2}-c_{2}(t)\right)_{+}+\kappa_{4} \min \left(c_{4}(t), s_{m}\right) \\
\dot{c}_{2}(t) & =-\kappa_{2} c_{2}(t)\left(l_{3}-c_{3}(t)\right)_{+}+\kappa_{1} c_{1}(t-\tau)\left(l_{2}-c_{2}(t-\tau)\right)_{+} \\
\dot{c}_{3}(t) & =-\kappa_{3} c_{3}(t)\left(l_{4}-c_{4}(t)\right)_{+}+\kappa_{2} c_{2}(t)\left(l_{3}-c_{3}(t)\right)_{+} \\
\dot{c}_{4}(t) & =-\kappa_{4} \min \left(c_{4}(t), s_{m}\right)+\kappa_{3} c_{3}(t)\left(l_{4}-c_{4}(t)\right)_{+} \\
\mathbf{c}(s) & =\boldsymbol{\Phi}(s), \quad s \in[0, \tau] .
\end{aligned}
$$

The BH limit theorem indicates that $\mathbf{C}(t)$ converges in probability to the solution of (12) as $N \rightarrow \infty$.

Remark 2. We remark that in the literature one often sees an arbitrarily chosen past history function for the delay differential equation. However, this does not make sense as the history function must depend on the dynamics of the given delay differential equation. If one knows when this process starts and the value where it starts with, then the method illustrated above provide a reasonable way to construct the past history function for delay differential equations with a fixed delay. Otherwise, one needs to collect experiment data and resort to parameter estimation methods to estimate this past history function. 


\subsection{Comparison of the stochastic model with a fixed delay and its corresponding deterministic system}

In this section we compare the results of the stochastic system with a fixed delay (9) to its corresponding deterministic system (in terms of number of pigs, i.e., $N \mathbf{c}(t)$ with $\mathbf{c}(t)$ being the solution to (12)). The stochastic system with a fixed delay (9) was simulated using Algorithm 2 in Section 2.2, and the deterministic system (12) was solved numerically using a linear spline approximation method (e.g., see [5, 6] for details).

All parameter values and initial conditions are taken from [4] and are given in Table 1 . The value of

\begin{tabular}{cll|cll}
\hline Parameters & Values & Units & Parameters & Values & Units \\
\hline$k_{1}$ & $1.674 / N$ & $1 /$ pigs days) & $\kappa_{1}$ & 1.674 & $1 /$ days \\
$k_{2}$ & $0.323 / N$ & $1 /$ pigs days) & $\kappa_{2}$ & 0.323 & $1 /$ days \\
$k_{3}$ & $4.521 / N$ & $1 /$ pigs days) & $\kappa_{3}$ & 4.521 & $1 /$ days \\
$k_{4}$ & 1 & $1 /$ days & $\kappa_{4}$ & 1 & $1 /$ days \\
\hline$L_{1}$ & $\infty$ & pigs & $l_{2}$ & $2.607 \cdot 10^{-1}$ & dimensionless \\
$L_{2}$ & $N \cdot 2.607 \cdot 10^{-1}$ & pigs & $l_{3}$ & $7.267 \cdot 10^{-1}$ & dimensionless \\
$L_{3}$ & $N \cdot 7.267 \cdot 10^{-1}$ & pigs & $l_{4}$ & $6.3 \cdot 10^{-3}$ & dimensionless \\
$L_{4}$ & $N \cdot 6.3 \cdot 10^{-3}$ & pigs & $s_{m}$ & $1.39 \cdot 10^{-1}$ & dimensionless \\
$S_{m}$ & $N \cdot 1.39 \cdot 10^{-1}$ & pigs & $c_{1}(0)$ & $2.528 \cdot 10^{-1}$ & dimensionless \\
\hline \hline$X_{1}(0)$ & {$\left[N \cdot 2.528 \cdot 10^{-1}\right]$} & pigs & $c_{2}(0)$ & $2.212 \cdot 10^{-1}$ & dimensionless \\
$X_{2}(0)$ & {$\left[N \cdot 2.212 \cdot 10^{-1}\right]$} & pigs & $c_{3}(0)$ & $4.739 \cdot 10^{-1}$ & dimensionless \\
$X_{3}(0)$ & {$\left[N \cdot 4.739 \cdot 10^{-1}\right]$} & pigs & $c_{4}(0)$ & $5.21 \cdot 10^{-2}$ & dimensionless \\
$X_{4}(0)$ & {$\left[N \cdot 5.21 \cdot 10^{-2}\right]$} & pigs & & &
\end{tabular}

Table 1: Parameter values and initial conditions for the stochastic and deterministic systems, where $N$ is the scaling parameter and $[z]$ denotes the integer closest to $z$.

the delay was set to be $\tau=5$. Two sample sizes were considered, $N=100$ and $N=10,000$, for the stochastic simulations. In Figure 1, the deterministic approximation $(N \mathbf{c}(t)$ with $\mathbf{c}(t)$ being the solution to (12)) is compared to five typical sample paths of the solution to the stochastic system (9). It is clear from this figure that the trajectories of the stochastic simulations follow the solution of its corresponding deterministic system, and the variance among sample paths of the stochastic solution decreases as the sample size increases. Figure 2 depicts the mean solution for the stochastic system (9) in comparison to the solution for the deterministic approximation $(N \mathbf{c}(t)$ with $\mathbf{c}(t)$ being the solution to (12)), where the mean solution was calculated by averaging 10,000 sample paths. We observe from this figure that as the sample size increases the mean solution of the stochastic system become closer to that of its corresponding deterministic system. 

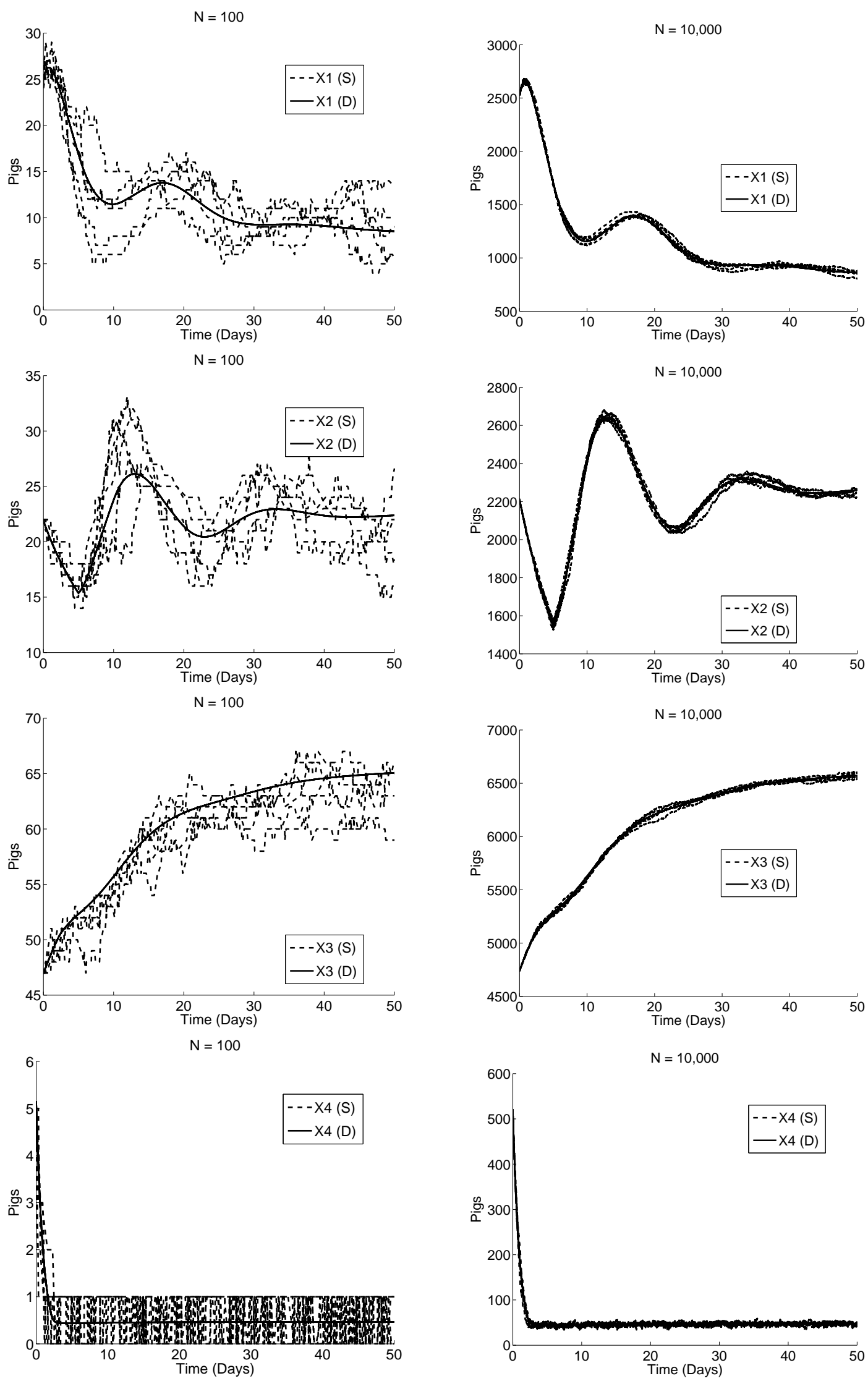

Figure 1: Results obtained by the stochastic system with a fixed delay $(\mathrm{S})$ and by the corresponding deterministic system (D) with $N=100$ (left column) and $N=10,000$ (right column). 

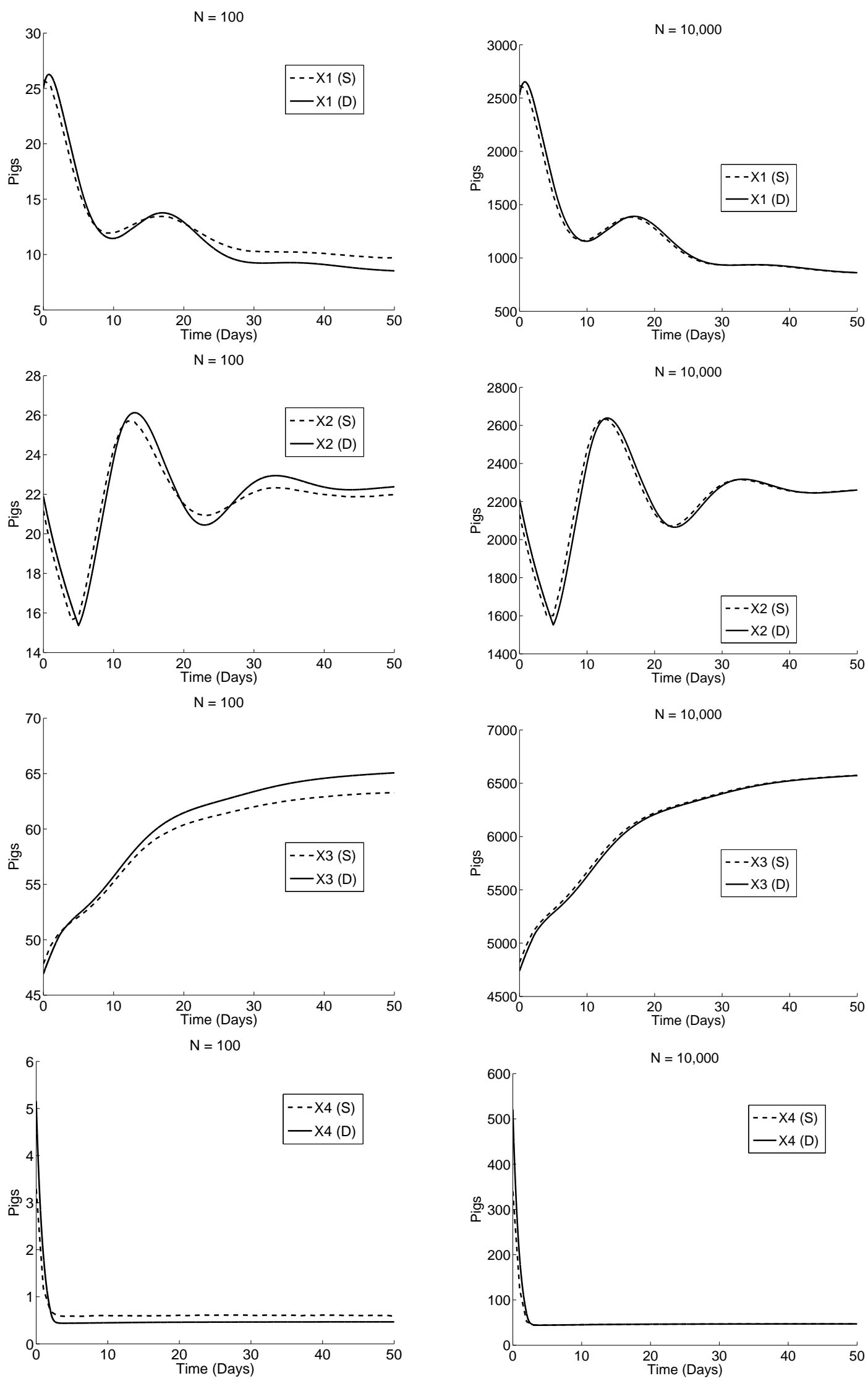

Figure 2: Results obtained by the stochastic system with a fixed delay $(\mathrm{S})$ and by the corresponding deterministic system (D) with $N=100$ (left column) and $N=10,000$ (right column). 


\section{The Pork Production Network Model With A Random Delay}

We next address the issue of how to implement random delays into the original pork production model. In the previous section we assumed that the delay was fixed. The interpretation of that formulation is that every transition from node 1 to node 2 is delayed by the same amount of time. We now want to consider the case where the amount of delayed time varies at each transition. The motivation for doing so is that in practice we would expect that the amount of time it takes to travel from node 1 to node 2 will vary based on a number of conditions, e.g., weather, traffic, road construction, etc. In this case it may not be a reasonable assumption that every transition is delayed by the same amount of time, but rather may vary for each transition that occurs. One way to implement this variation of delay times is to consider the delay to be a random variable that will be sampled for each transition. The resulting system will be called the stochastic model with a random delay. For this model, we again assume that all transitions occur instantaneously except for the arrival of pigs transitioning from node 1 to 2 , and the process starts from $t=0$, that is, $\mathbf{X}(t)=0$ for $t<0$.

\subsection{Algorithms for stochastic systems with random delays}

Roussel and Zhu propose a method in [24] for simulating a stochastic system with random delays. Specifically, the algorithm for a stochastic system with random delays can be implemented in a way that is a slight modification of Algorithm 2 in Section 2.2. The algorithm simulates which reaction will take place next. In the case of fixed delays, if a delayed transition fires, then the effect of that transition is held until the specified amount of time passes. In the case of random delays, when a delayed transition occurs, the delay is sampled from a given distribution, and this value is taken as the amount of time that must pass before the transition affects the system. Each time a delayed transition is fired, a new value of the delay is drawn from the given distribution. The full details of the algorithm are outlined below.

When a delayed reaction fires, one draws a random number from the desired distribution to use for the delay. Previously, in the fixed delay case, all that was needed was to store the delayed time in the array $\mathbf{m}_{l}$ in the second to last position. This guaranteed that the array $\mathbf{m}_{l}$ was sorted in ascending order (recall that the last element in $m_{l}$ was initialized to infinity). If the delay is random we cannot take this approach, instead the delayed time must be stored in $\mathbf{m}_{l}$ (in any position) and then $\mathbf{m}_{l}$ must be sorted in ascending order. Let us again assume that the stochastic system has $M$ transitions with transition rates $\lambda_{i}(\mathbf{x})$ for $i=1,2, \ldots, M$. In addition, if the $j$ th transition is a delayed one, then we let $\tau_{j}$ denote the delay time between the initiation and completion for this transition, where its value is sampled from a given distribution at each time this delayed transition is fired.

\section{Algorithm 3: Modified Next Reaction Method For Stochastic Systems With Random Delays}

1. Set the initial condition for each state for $t=0$. Set $T_{i}=0$, and array $\mathbf{m}_{i}=(\infty)$ for $i=1,2, \ldots, M$.

2. Calculate each transition rate $\lambda_{i}$ at the given state for each $i=1,2, \ldots, M$.

3. Generate $M$ independent, uniform $(0,1)$ random numbers $r_{i}$.

4. Set $P_{i}=\ln \left(1 / r_{i}\right)$ for $i=1,2, \ldots, M$.

5. Set $\Delta t_{i}=\left(P_{i}-T_{i}\right) / \lambda_{i}$ for $i=1,2, \ldots, M$.

6. Set $\Delta=\min _{1 \leq i \leq M}\left(\Delta t_{i}, \mathbf{m}_{i}(1)-t\right)$.

7. Set $t=t+\Delta$.

8. If $\Delta t_{l}$ obtained the minimum in step 6 then do the following. If the $l$ reaction is a $\mathrm{ND}$, then update the system according to the reaction $l$. If reaction $l$ is a CD then store the time $t+\tau_{l}$ in $\mathbf{m}_{l}$ and sort $\mathbf{m}_{l}$ in ascending order, where $\tau_{l}$ is sampled from a given distribution. If reaction $l$ is a ICD then update the system according to the initiation of the $l$ reaction and store $t+\tau_{l}$ in $\mathbf{m}_{l}$ and then sort $\mathbf{m}_{l}$ in ascending order, where $\tau_{l}$ is sampled from a given distribution.

9. If $\mathbf{m}_{l}(1)-t$ obtained the minimum in step 6 then update the system according the completion of reaction $l$ and delete the first element in $\mathbf{m}_{l}$.

10. Set $T_{i}=T_{i}+\lambda_{i} \Delta$ for $i=1, \ldots, M$.

11. For the transition $l$ which initiated, choose a uniform random number $(0,1), r$, and set $P_{l}=P_{l}+\ln (1 / r)$.

12. Recalculate each transition rate $\lambda_{i}$ at the given new state for $i=1,2, \ldots, M$.

13. Return to step 5 . 


\subsection{The corresponding deterministic system for the stochastic model with a random delay}

In [26], Schlicht and Winkler showed that if all the transition rates are linear, then the mean solution of the stochastic system with random delays can be described by a system of deterministic differential equations with a distributed delay, where the delay kernel is the probability density function of the given distribution for the random delay. Even though the transition rates in our pork production model are nonlinear, we still would like to explore whether or not such a deterministic system can be used as a possible corresponding deterministic system for our stochastic system with a random delay and explore the relationship between them.

Let $G(t)$ be the probability density function of the random delay. Then the corresponding deterministic system for our stochastic system with delay is given by

$$
\begin{aligned}
& \dot{c}_{1}(t)=-\kappa_{1} c_{1}(t)\left(l_{2}-c_{2}(t)\right)_{+}+\kappa_{4} \min \left(c_{4}(t), s_{m}\right) \\
& \dot{c}_{2}(t)=-\kappa_{2} c_{2}(t)\left(l_{3}-c_{3}(t)\right)_{+}+\int_{-\infty}^{t} G(t-s) \kappa_{1} c_{1}(s)\left(l_{2}-c_{2}(s)\right)_{+} d s \\
& \dot{c}_{3}(t)=-\kappa_{3} c_{3}(t)\left(l_{4}-c_{4}(t)\right)_{+}+\kappa_{2} c_{2}(t)\left(l_{3}-c_{3}(t)\right)_{+} \\
& \dot{c}_{4}(t)=-\kappa_{4} \min \left(c_{4}(t), s_{m}\right)+\kappa_{3} c_{3}(t)\left(l_{4}-c_{4}(t)\right)_{+} \\
& c_{i}(0)=c_{i 0}, \quad i=1,2,3,4, \\
& c_{i}(s)=0, \quad s<0, \quad i=1,2,3,4,
\end{aligned}
$$

We note that numerically solving a system of the form (13) may prove to be difficult due to the distributed delay term. However, if we make additional assumptions on the delay kernel, we can transform a system with a distributed delay into a system of ODE's. Specifically, if we assume that the delay kernel has the form

$$
G(u ; \alpha, n)=\frac{\alpha^{n} u^{n-1} e^{-\alpha u}}{(n-1) !}
$$

with $\alpha>0$ and $n$ being a positive integer number, that is, $G$ is the probability density function of a Gamma distributed random variable with mean being $n / \alpha$ and the variance being $n / \alpha^{2}$, then by way of the linear chain trick [13] (e.g., see [8, 18, 25] and the references therein) we can transform the system (13) into a system of ODE's. For example, for the case $n=1$, if we let $c_{5}(t)=\int_{-\infty}^{t} \alpha e^{-\alpha(t-\theta)} \kappa_{1} c_{1}(\theta)\left(l_{2}-c_{2}(\theta)\right)_{+} d \theta$, then this substitution yields the system of ODE's

$$
\begin{aligned}
& \dot{c}_{1}(t)=-\kappa_{1} c_{1}(t)\left(l_{2}-c_{2}(t)\right)_{+}+\kappa_{4} \min \left(c_{4}(t), s_{m}\right) \\
& \dot{c}_{2}(t)=-\kappa_{2} c_{2}(t)\left(l_{3}-c_{3}(t)\right)_{+}+c_{5}(t) \\
& \dot{c}_{3}(t)=-\kappa_{3} c_{3}(t)\left(l_{4}-c_{4}(t)\right)_{+}+\kappa_{2} c_{2}(t)\left(l_{3}-c_{3}(t)\right)_{+} \\
& \dot{c}_{4}(t)=-\kappa_{4} \min \left(c_{4}(t), s_{m}\right)+\kappa_{3} c_{3}(t)\left(l_{4}-c_{4}(t)\right)_{+} \\
& \dot{c}_{5}(t)=\alpha \kappa_{1} c_{1}(t)\left(l_{2}-c_{2}(t)\right)_{+}-\alpha c_{5}(t) \\
& c_{i}(0)=c_{i 0}, \quad i=1,2,3,4, \\
& c_{5}(0)=\int_{-\infty}^{0} \alpha e^{\alpha \theta} \kappa_{1} c_{1}(\theta)\left(l_{2}-c_{2}(\theta)\right)_{+} d \theta .
\end{aligned}
$$

which is equivalent to (13).

The advantage of using this linear chain trick is two fold. The resulting system of ODE's is much easier to solve compared to the system (13) where there is a distributed delay. In addition, we can use the Kurtz's limit theorem to construct a corresponding stochastic system which converges to the resulting system of ODE's. This approach will be considered in Section 3.4.

\subsection{Comparison of the stochastic model with a random delay and its corre- sponding deterministic system}

All parameter values and initial conditions are taken as in Section 2 (Table 1). For the probability density function $G(u ; \alpha, n), n$ was taken to be 1 and $\alpha$ to be 0.2 , which implies that the mean value of the random delay is 5 and its variance is 25.0. Sample sizes of $N=100$ and $N=10,000$ were considered for the stochastic system with a random delay. As before, the stochastic system was simulated for 10,000 trials, and the mean solution was computed. 
Figure 3 compares the solution of deterministic system (in terms of number of pigs, i.e., $N \mathbf{c}(t)$ with $\mathbf{c}(t)$ being the solution to (15)) and five typical sample paths of the solution to the stochastic system with a random delay. We observe from this figure that the trajectories of the stochastic simulations follow the
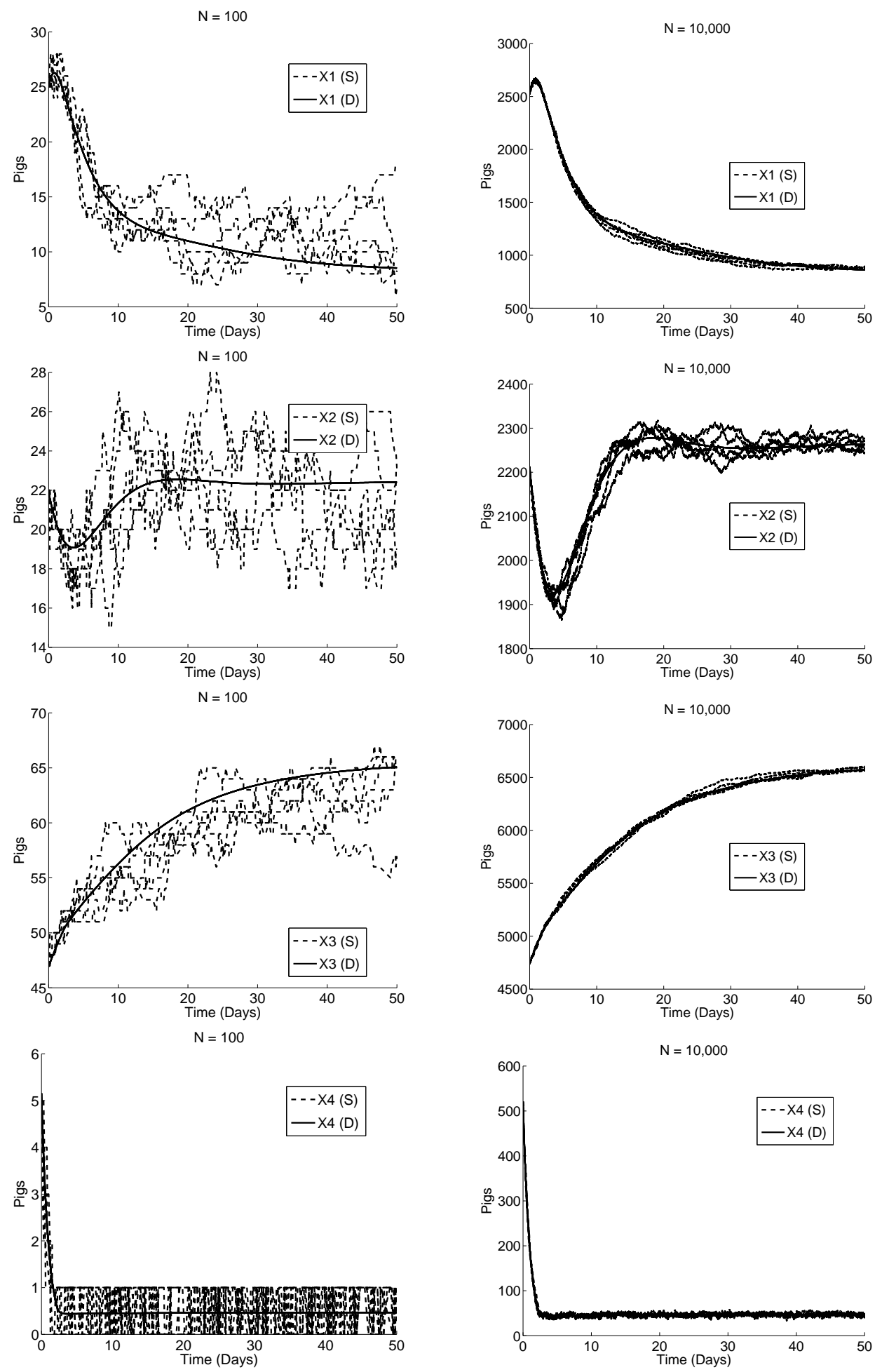

Figure 3: Results obtained by the stochastic system with a random delay $(\mathrm{S})$ and by the corresponding deterministic system (D) with $N=100$ (left column) and $N=10,000$ (right column). 
solution of the deterministic system, and the variation of the sample paths of the solution to the stochastic system with a random delay decreases as the sample size increases. Figure 4 depicts the mean solution for the stochastic system with a random delay in comparison to the solution of deterministic system (in terms of number of pigs). It is seen from this figure that as the sample size increases the mean solution of the stochastic
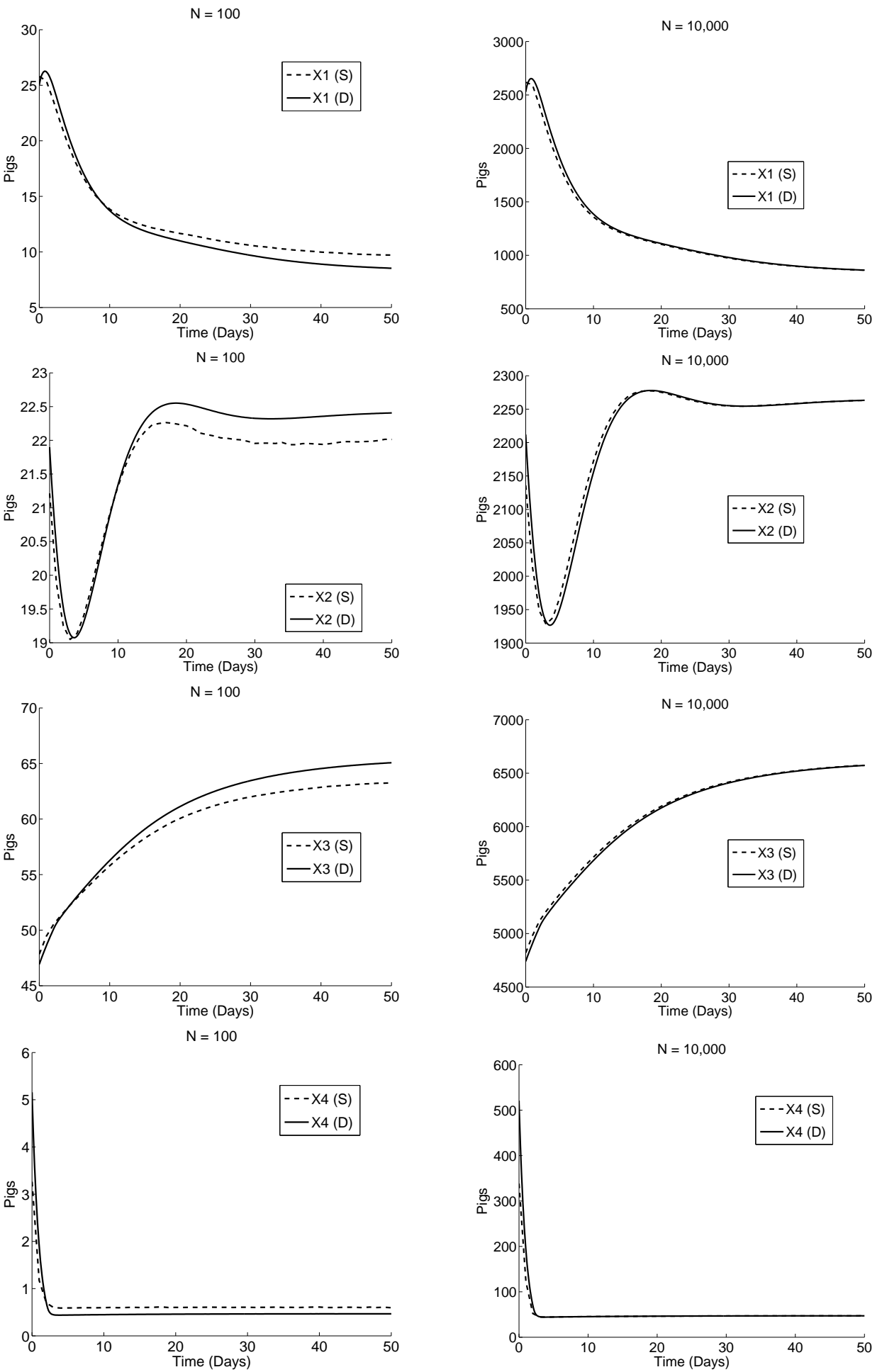

Figure 4: Results obtained by the stochastic system with a random delay (S) and by the corresponding deterministic system (D) with $N=100$ (left column) and $N=10,000$ (right column). 
system with a random delay become closer to the solution of deterministic system. Hence, not only are the sample paths of the solution to stochastic system with a random delay showing less variation for larger sample sizes, but the expected value of the solution is better approximated by the solution of deterministic system for large sample sizes. Thus, the deterministic system (15) (or the deterministic differential equation with a distributed delay (13)) could be used to serve as a reasonable corresponding deterministic system for this particular stochastic system with a random delay (with the given parameter values and initial conditions).

\subsection{The corresponding constructed stochastic system for the system of ODE's} (15)

Based on the Kurtz's limit theorem, one can construct a stochastic system (without delays) which will converge to the system of ODE's given in (15). Let $\mathbf{e}_{i} \in \mathbb{Z}^{5}$ be the $i$ th unit column vector, $i=1,2, \ldots, 5$. The transition rate $\lambda_{j}(\mathbf{x})$ and the corresponding state change vector $\mathbf{v}_{j}$ at the $j$ th transition, $j=1,2, \ldots, 7$, are given by

$$
\begin{array}{lll}
\lambda_{1}(\mathbf{x})=k_{1} x_{1}\left(L_{2}-x_{2}\right)_{+}, & & \mathbf{v}_{1}=-\mathbf{e}_{1}, \\
\lambda_{2}(\mathbf{x})=k_{2} x_{2}\left(L_{3}-x_{3}\right)_{+}, & & \mathbf{v}_{2}=-\mathbf{e}_{2}+\mathbf{e}_{3}, \\
\lambda_{3}(\mathbf{x})=k_{3} x_{3}\left(L_{4}-x_{4}\right)_{+}, & \mathbf{v}_{3}=-\mathbf{e}_{3}+\mathbf{e}_{4}, \\
\lambda_{4}(\mathbf{x})=k_{4} \min \left(x_{4}, S_{m}\right), & & \mathbf{v}_{4}=-\mathbf{e}_{4}+\mathbf{e}_{1} \\
\lambda_{5}(\mathbf{x})=x_{5}, & & \mathbf{v}_{5}=\mathbf{e}_{2} \\
\lambda_{6}(\mathbf{x})=\alpha x_{5}, & & \mathbf{v}_{6}=-\mathbf{e}_{5} \\
\lambda_{7}(\mathbf{x})=\alpha k_{1} x_{1}\left(L_{2}-x_{2}\right)_{+}, & \mathbf{v}_{7}=\mathbf{e}_{5} .
\end{array}
$$

Then by (3) we know that the stochastic system corresponding to the above transitions rates and state change vectors is given by

$$
\begin{aligned}
& X_{1}(t)=X_{1}(0)-Y_{1}\left(\int_{0}^{t} \lambda_{1}(\mathbf{X}(s)) d s\right)+Y_{4}\left(\int_{0}^{t} \lambda_{4}(\mathbf{X}(s)) d s\right) \\
& X_{2}(t)=X_{2}(0)-Y_{2}\left(\int_{0}^{t} \lambda_{2}(\mathbf{X}(s)) d s\right)+Y_{5}\left(\int_{0}^{t} \lambda_{5}(\mathbf{X}(s)) d s\right) \\
& X_{3}(t)=X_{3}(0)-Y_{3}\left(\int_{0}^{t} \lambda_{3}(\mathbf{X}(s)) d s\right)+Y_{2}\left(\int_{0}^{t} \lambda_{2}(\mathbf{X}(s)) d s\right) \\
& X_{4}(t)=X_{4}(0)-Y_{4}\left(\int_{0}^{t} \lambda_{4}(\mathbf{X}(s)) d s\right)+Y_{3}\left(\int_{0}^{t} \lambda_{3}(\mathbf{X}(s)) d s\right) \\
& X_{5}(t)=X_{5}(0)-Y_{6}\left(\int_{0}^{t} \lambda_{6}(\mathbf{X}(s)) d s\right)+Y_{7}\left(\int_{0}^{t} \lambda_{7}(\mathbf{X}(s)) d s\right) .
\end{aligned}
$$

By the Kurtz's limit theorem, we know that $\left(\frac{X_{1}(t)}{N}, \ldots, \frac{X_{5}(t)}{N}\right)^{T}$ will converge to the solution of the ODE system (15).

Recall that for the deterministic differential equation with a distributed delay, one assumes that each individual is different and may take different time to progress from one node to another so that for a large population one can use a distributed delay to approximate it. Hence, for the stochastic system (16) constructed from a deterministic differential equation with a distributed delay, each individual may also be treated differently. We remark that even though the constructed stochastic system (16) may have no biological meaning, it will be used to serve as a comparison for the stochastic system with a random delay. The reason we do this is that the stochastic system (16) is constructed based on Kurtz's limit theorem from the system of ODE's (15), which is also used as a possible corresponding deterministic system for the stochastic system with a random delay (as we demonstrated in the previous section).

\subsection{Comparison of the constructed stochastic system (16) and its corresponding deterministic system}

All parameter values and initial conditions remain as in Section 2 (Table 1). The mean value of the random delay was again set to be $\tau=5$ and recall that $\alpha=0.2$. The stochastic system was simulated using Algorithm 1 in Section 1.2 with sample sizes of $N=100, N=10,000$ and $N=100,000$. As before, the stochastic 
system was simulated for 10,000 trials for each sample size, and the mean solution was then computed by averaging these 10,000 sample paths.

Figure 5 depicts five typical sample paths of the solution to the stochastic system (16) compared to the solution to the corresponding deterministic system (in terms of number of pigs, i.e., $N \mathbf{c}(t)$ with c $(t)$ being the solution to (15)). While Figure 6 shows the mean solution to the stochastic system (16) in comparison
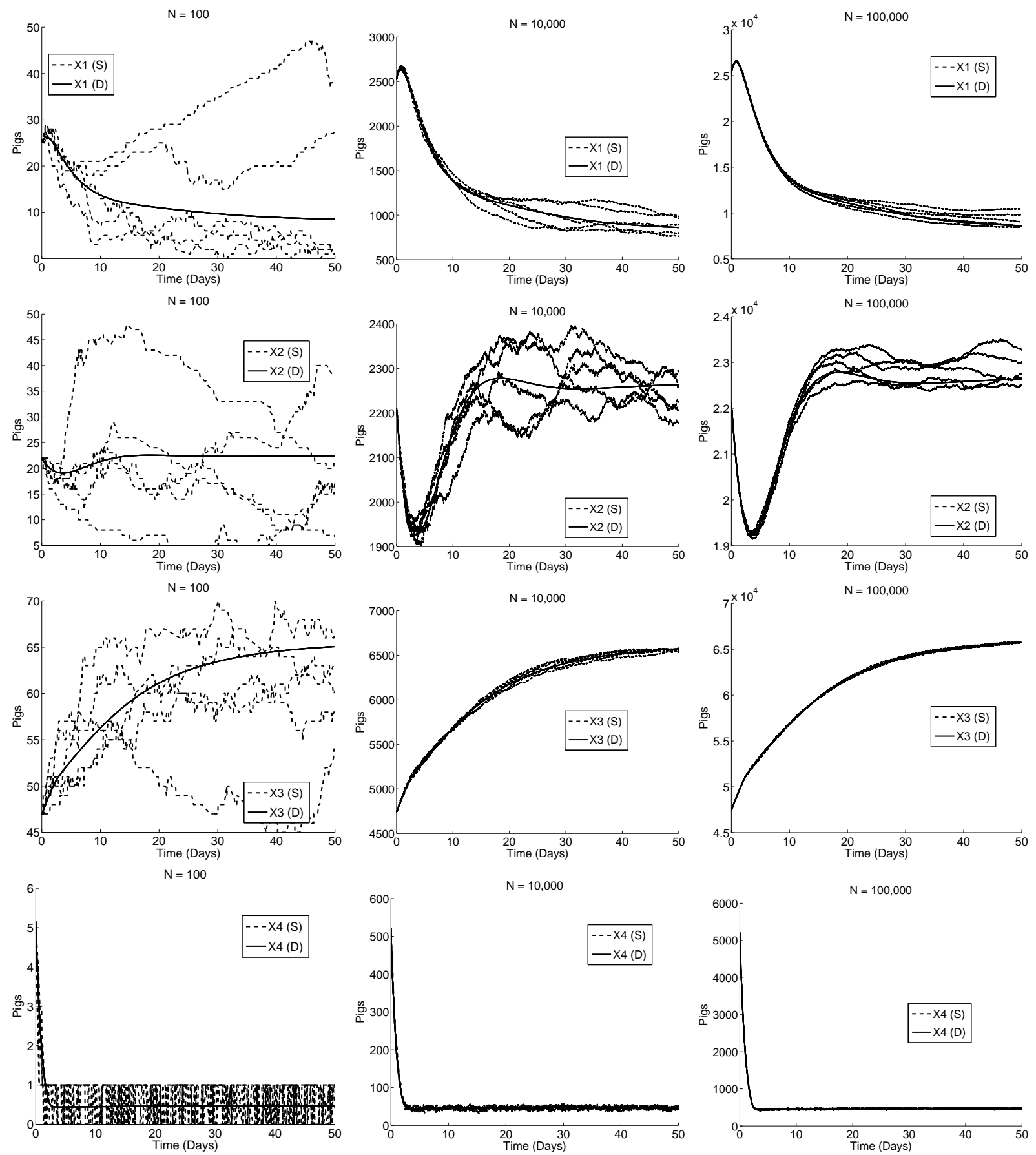

Figure 5: Results obtained for the deterministic system (D) and the corresponding constructed stochastic system (S) with a sample size of $N=100$ (left column), $N=10,000$ (middle column), and $N=100,000$ (right column).

to the solution to the corresponding deterministic system. We observe from these two figures that, as might 

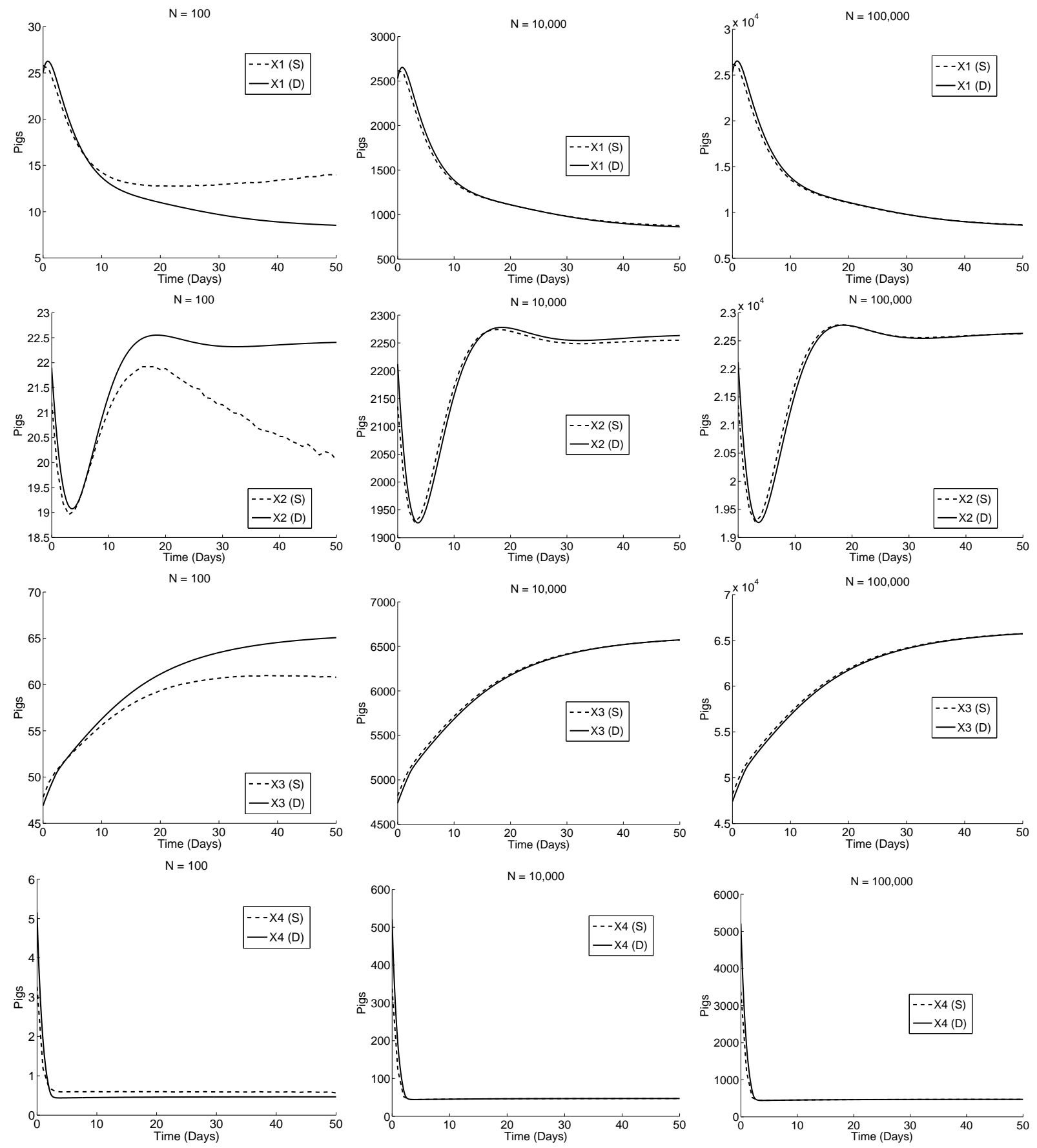

Figure 6: Results obtained for the deterministic system (D) and the corresponding constructed stochastic system (S) with a sample size of $N=100$ (left column), $N=10,000$ (middle column), and $N=100,000$ (right column).

be expected, the variation of the sample paths of the solution to stochastic system (16) decreases as the sample size increases, and its mean solution become closer to the solution of its corresponding deterministic system as the sample size increases. It is also noted that for each of the same sample sizes $N$, the variation of sample paths is much higher compared to those of the stochastic system with a random delay, especially for node 1 and node 2 . 


\subsection{Comparison of the stochastic system with a random delay and the con- structed stochastic system}

The numerical results in the previous section demonstrate that with the same sample size the sample paths of the solutions to the stochastic system with a random delay have less variation than those obtained for the corresponding constructed stochastic system (16). In this section we make a further comparison of these two stochastic systems and explore their relationship with each other.

For all the simulations below, the model parameter values and initial conditions remain as in Section 2 (Table 1). In addition, the random delay is assumed to be Gamma distributed with probability density function $G(u ; \alpha, n)$ as defined in (14), where the expected value of the random delay is always chosen to be 5 (i.e., $n / \alpha=5$ ). Each stochastic system was simulated 10,000 times with various sample sizes. For each of the sample sizes, a histogram was constructed for $X_{i}(t), i=1,2,3,4$, at $t=25$ and at $t=50$, for each stochastic system based on these 10,000 sample paths.

\subsubsection{The effect of sample size $N$ on the comparison of these two stochastic systems}

In this section, we investigate the effect of the sample size $N$ on the comparison of the stochastic system with a random delay and the constructed stochastic system (16), where $G(u ; \alpha, n)$ is chosen with $n=1$ and $\alpha=0.2$ as before.

Using the same sample size for these two stochastic systems: In this case, we simulated each stochastic system 10,000 times with a sample size of $N=100, N=1,000$, and $N=10,000$. Figure 7 depicts the histograms of each node for these two stochastic systems with $N=100$ (left column), $N=1,000$ (middle column) and $N=10,000$ (right column) at time point $t=25$, and Figure 8 illustrates these histograms at $t=50$. We observe from these two figures that the histograms for each of the stochastic systems do not match well except for in the case of $X_{4}$, which is probably because the delay only occurs from node 1 to node 2 , and hence it has less effect on $X_{4}$ than on all the other nodes due to the movement from one node to the next occurring only in the forward direction. Specifically, it is seen that for all the sample sizes investigated, the histogram plots of $X_{1}, X_{2}, X_{3}$ obtained for the constructed stochastic system (16) appear more dispersed than those for the stochastic system with a random delay, and this is especially obvious for $X_{1}$ and $X_{2}$. As we remarked earlier that individuals in the same node for the constructed stochastic system (16) may be treated differently, while individuals for the stochastic system with a random delay are treated the same. This means that the constructed stochastic system is more "random" than the stochastic system with a random delay, and thus it shows more variation. Figures 7 and 8 also reveal that the histogram plots of $X_{1}, X_{2}, X_{3}$ obtained for the stochastic system with a random delay are symmetric for all the sample sizes investigated, while those for the constructed stochastic system (16) are asymmetric when the sample size is small, but becomes more symmetric as the sample size increases. 

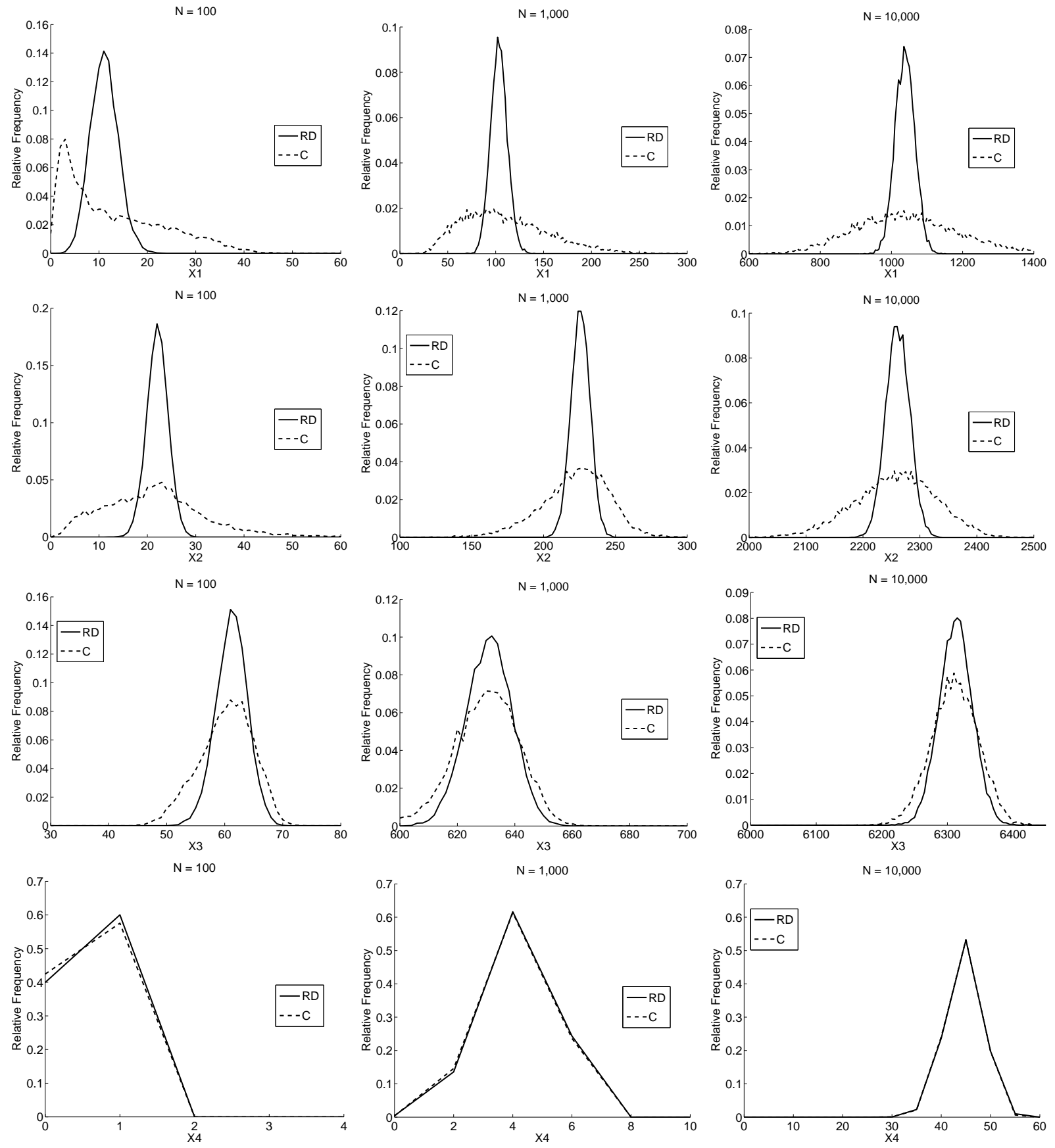

Figure 7: Histograms of the stochastic system with random delays (RD) and the constructed stochastic system (C) with $N=100$ (left column), $N=1,000$ (middle column), and $N=10,000$ (right column) at $t=25$. 

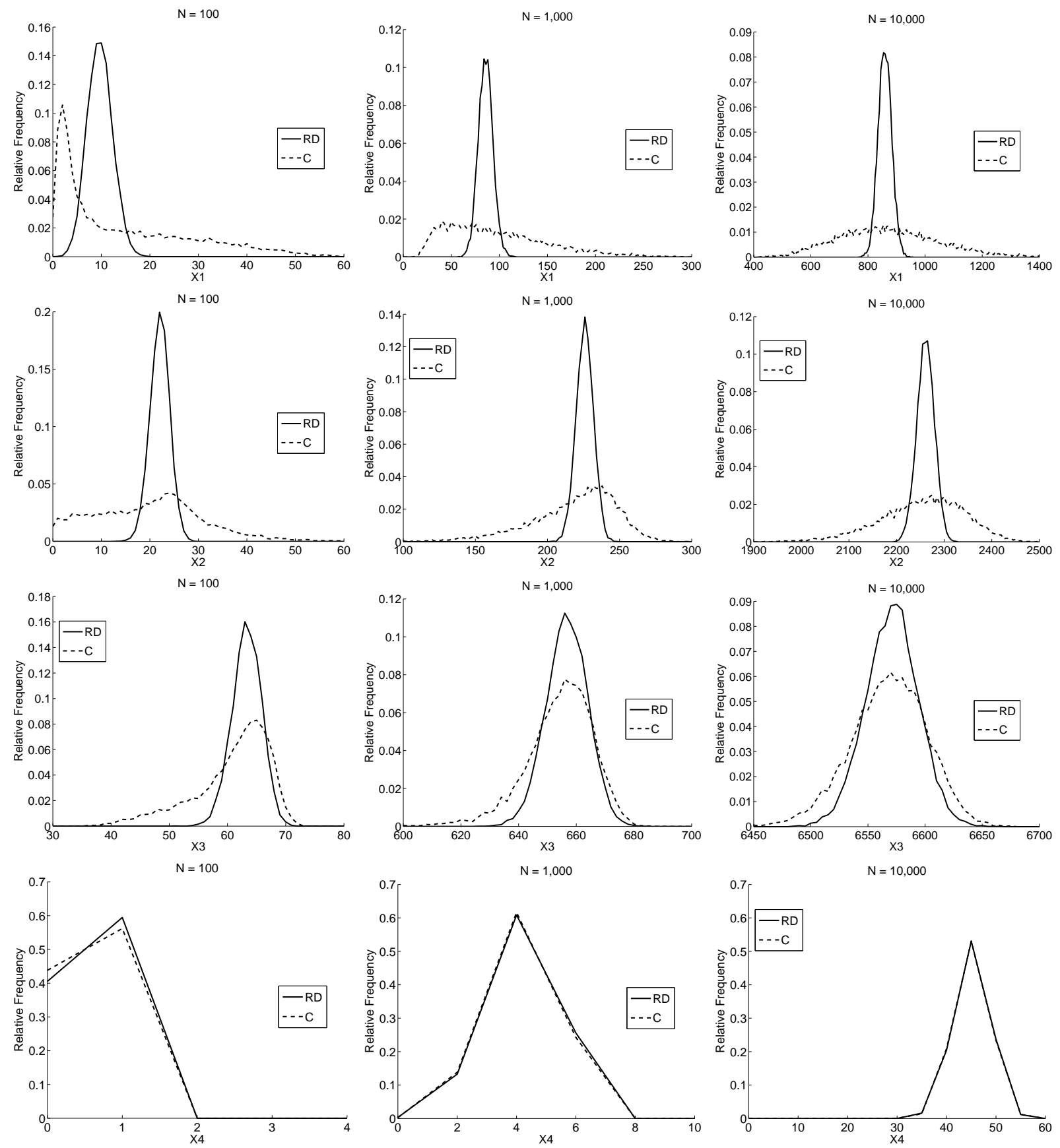

Figure 8: Histograms of the stochastic system with random delays (RD) and the constructed stochastic system (C) with $N=100$ (left column), $N=1,000$ (middle column), and $N=10,000$ (right column) at $t=50$. 
Using different sample size for these two stochastic systems: In Figure 9, we compare the histogram plots obtained from the stochastic system with a random delay for a sample size of $N=10,000$ to those obtained from the constructed stochastic system with a sample size of $N=100,000$ and we see that the two histograms are in better agreement for nodes 1 and 2. We offer one possible explanation for this occurrence.
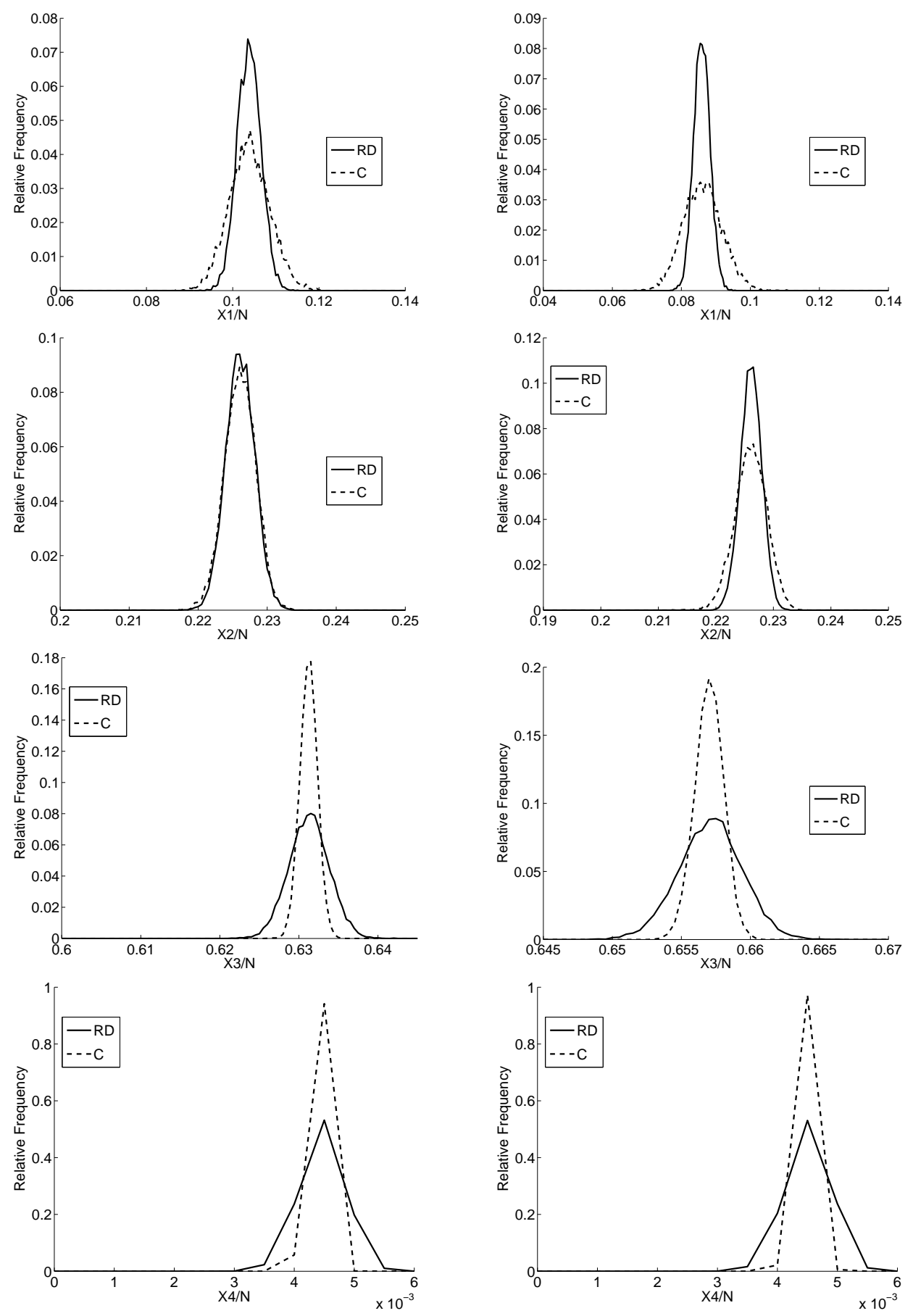

Figure 9: Histograms of the stochastic system with random delays (RD) with a sample size of $N=10,000$ and the constructed stochastic system with a sample size of $N=100,000$ at $t=25$ (left column) and $t=50$ (right column). The histograms are plotted with respect to density.

Both stochastic systems can be approximated by the same deterministic system (with the given parameter values and initial conditions), yet it is possible that the two stochastic systems are "converging" to the 
deterministic solution at different rates, thus different values for sample size are need to obtain the same order of approximation.

\subsubsection{The effect of the variance of a random delay on the comparison of these two stochastic systems}

What remains to be investigated is how the variance of the random delay affects the comparison of the two stochastic systems. However, in order to change the variance, the shape and rate parameters $n$ and $\alpha$ in the probability density function $G(u ; \alpha, n)$ for the random delay must be altered in order to keep the expected value of the random delay to be the same. Specifically, the value of $n$ determines the number of additional equations to reduce the deterministic system with a distributed delay into a system of ODE's. Thus, for each additional variance to be considered, a new system of ODE's and the corresponding stochastic system must be derived.

For the case where $n=10$ and $\alpha=2$, we have a mean of 5 and a variance of 2.5 for the random delay. Now using the substitutions

$$
c_{j+4}(t)=\int_{-\infty}^{t} \frac{\alpha^{j}(t-\theta)^{j-1}}{(j-1) !} e^{-\alpha(t-\theta)} \kappa_{1} c_{1}(\theta)\left(l_{2}-c_{2}(\theta)\right)_{+} d \theta, \quad j=1,2,3, \ldots, 10,
$$

we obtain the following system of ODE's that is equivalent to (13) with delay kernel being $G(u ; 2,10)$

$$
\begin{aligned}
\dot{c}_{1}(t) & =-\kappa_{1} c_{1}(t)\left(l_{2}-c_{2}(t)\right)_{+}+\kappa_{4} \min \left(c_{4}(t), s_{m}\right) \\
\dot{c}_{2}(t) & =-\kappa_{2} c_{2}(t)\left(l_{3}-c_{3}(t)\right)_{+}+c_{14}(t) \\
\dot{c}_{3}(t) & =-\kappa_{3} c_{3}(t)\left(l_{4}-c_{4}(t)\right)_{+}+\kappa_{2} c_{2}(t)\left(l_{3}-c_{3}(t)\right)_{+} \\
\dot{c}_{4}(t) & =-\kappa_{4} \min \left(c_{4}(t), s_{m}\right)+\kappa_{3} c_{3}(t)\left(l_{4}-c_{4}(t)\right)_{+} \\
\dot{c}_{5}(t) & =\alpha \kappa_{1} c_{1}(t)\left(l_{2}-c_{2}(t)\right)_{+}-\alpha c_{5}(t) \\
\dot{c}_{i}(t) & =\alpha c_{i-1}-\alpha c_{i} \quad \text { for } i=6,7, \ldots, 14 \\
c_{i}(0) & =c_{i 0}, \quad i=1,2,3,4 \\
c_{j+4}(0) & =\int_{-\infty}^{0} \frac{\alpha^{j}(-\theta)^{j-1}}{(j-1) !} e^{\alpha \theta} \kappa_{1} c_{1}(\theta)\left(l_{2}-c_{2}(\theta)\right)_{+} d \theta, \quad j=1,2,3, \ldots, 10 .
\end{aligned}
$$

We can construct the stochastic system which will converge to this ODE system in the same manner as before. To avoid confusion we will refer to this new stochastic system as the constructed stochastic system with $\alpha=2$ to distinguish it from the previously constructed system in which we had $\alpha=0.2$.

The constructed stochastic system with $\alpha=2$ was simulated 10,000 times with a sample size of $N=100$ and $N=1,000$. The stochastic system with a random delay was simulated for 10,000 trials with $N=100$ and $N=1,000$, where the random delay has the probability density function $G(u ; \alpha, n)$ with $n=10$ and $\alpha=2$. The resulting histograms are shown in Figures 10 and 11. From here it is seen that there is much more agreement between the histograms than the corresponding ones in Section 3.6.1 when the variance was 25.0. In addition, we observe that the histograms of the constructed system with $\alpha=2$ are a little closer to the histograms to the stochastic system with a random delay for the larger sample size. Overall, we see that the variance of the random delay has a decided effect on the agreement between these two stochastic systems. 

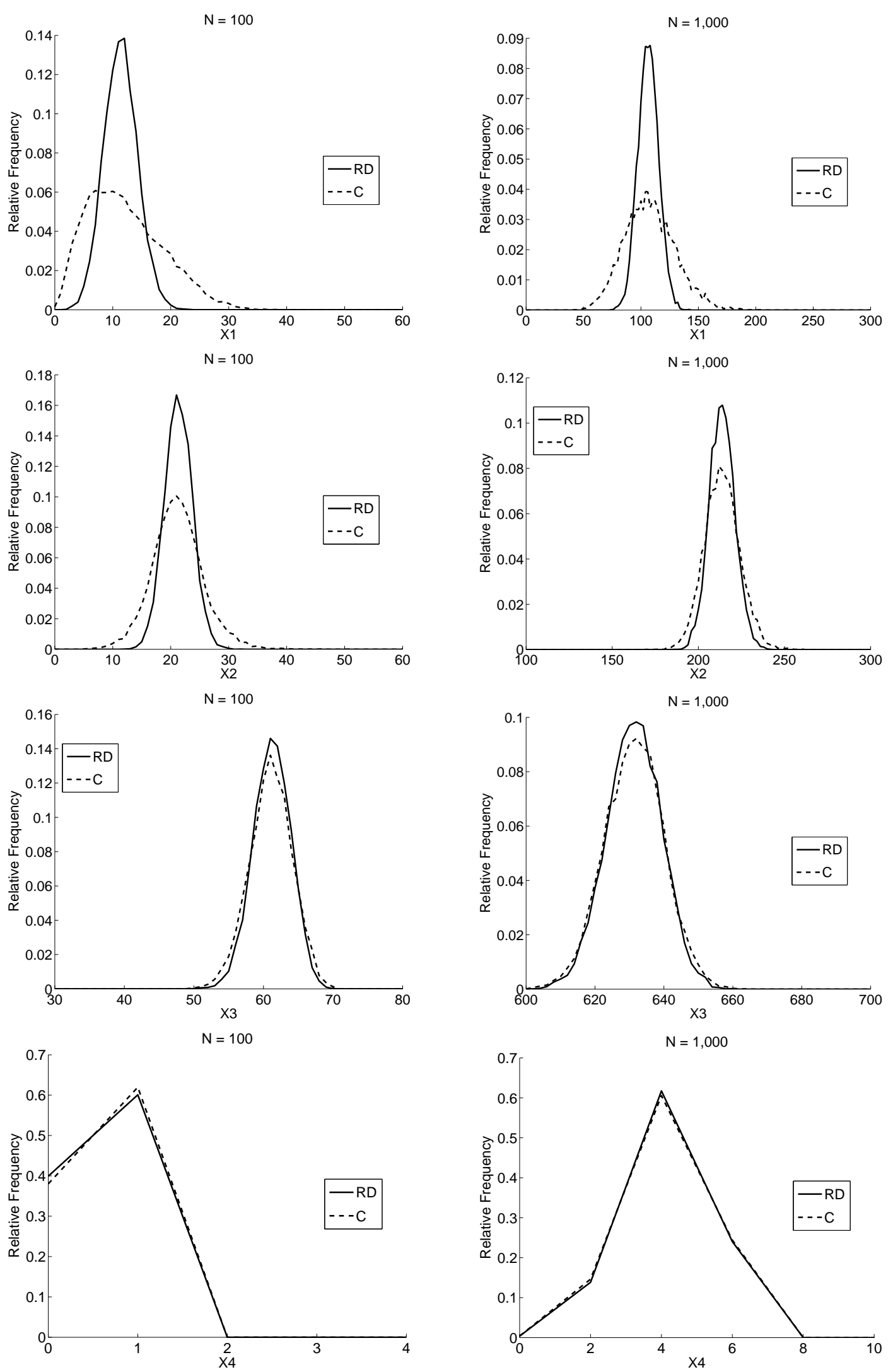

Figure 10: Histograms of the stochastic system with random delays (RD) and the constructed stochastic system (C) with $N=100$ (left column), $N=1,000$ (right column) at $t=25$. The random delay was chosen from a gamma distribution with a mean of 5 and variance of 2.5 . 

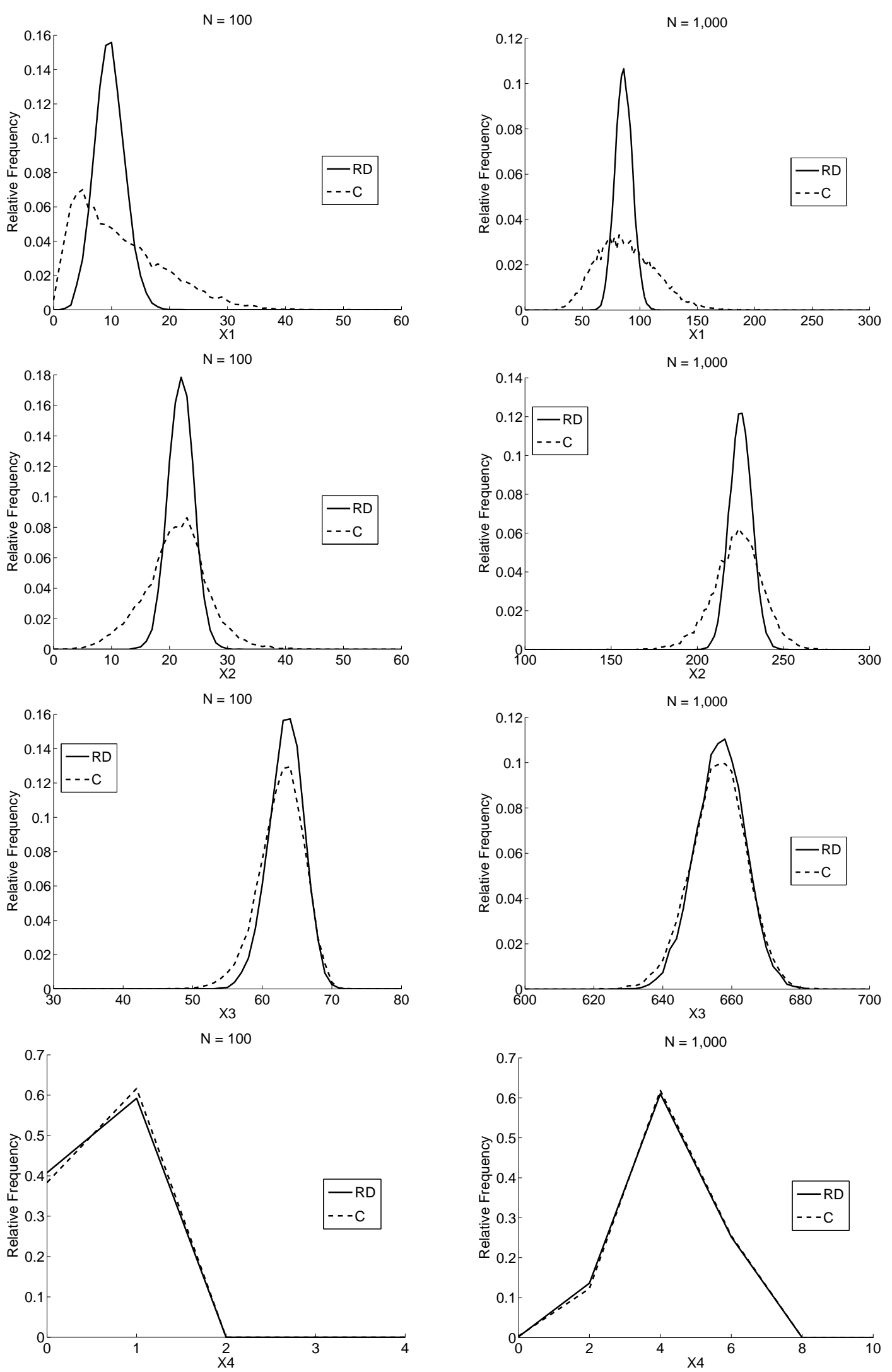

Figure 11: Histograms of the stochastic system with random delays (RD) and the constructed stochastic system (C) with $N=100$ (left column), $N=1,000$ (right column) at $t=50$. The random delay was chosen from a gamma distribution with a mean of 5 and variance of 2.5 . 


\section{Comparison Of The Pork Production Model With A Fixed De- lay And One With A Random Delay}

In this section we compare the pork production model with a fixed delay as in Section 2, to the one with a random delay in Section 3. The value of the delay for the stochastic system with a fixed delay was chosen to be the expected value of the random delay in the stochastic system with a random delay, where the random delay is assumed to be Gamma distributed with probability density function $G(u ; \alpha, n)$ as defined in (14).

For all the simulations below, the model parameter values and initial conditions remain as in Section 2 (Table 1), and the expected value of the random delay is chose to be 5 (i.e., $n / \alpha=5$ ). Each stochastic system was simulated 10,000 times with a sample size of $N=100, N=1,000$, and $N=10,000$. For each of the sample sizes, a histogram was constructed for $X_{i}(t), i=1,2,3,4$, at $t=25$ and $t=50$, for each stochastic system based on these 10,000 sample paths.

\subsection{The case with the variance of the random delay $\sigma^{2}=25$}

As a first consideration, the variance of the random delay is chosen to be 25.0 , that is, $n / \alpha^{2}=25.0$. In this case the probability density function $G(u ; \alpha, n)$ for the random delay is chosen such that $n=1$ and $\alpha=0.2$.

Figure 12 depicts the histograms for these two stochastic systems with $N=100$ (left column), $N=1,000$ (middle column), and $N=10,000$ (right column) at $t=25$. While Figure 13 illustrates these histograms at $t=50$. We observe from these two figures that the histograms agree well in all cases for nodes 1,3 and 4 , and they agree reasonably well for $X_{2}(t)$ at $t=50$ for all the sample sizes considered. However, for $X_{2}(t)$ at $t=25$ there are larger differences between these two stochastic systems, and the histograms actually deviate increasingly as the sample size $N$ is increased. Specifically, the histograms for the stochastic system with a fixed delay was shifted more to the left side as the sample size increases compared to the corresponding ones for the stochastic system with a random delay. But for these cases, the histograms of $X_{2}(t)$ at $t=25$ obtained for stochastic system with a fixed delay still have similar unimodal shape and dispersion as the corresponding ones obtained for the stochastic system with a random delay. 

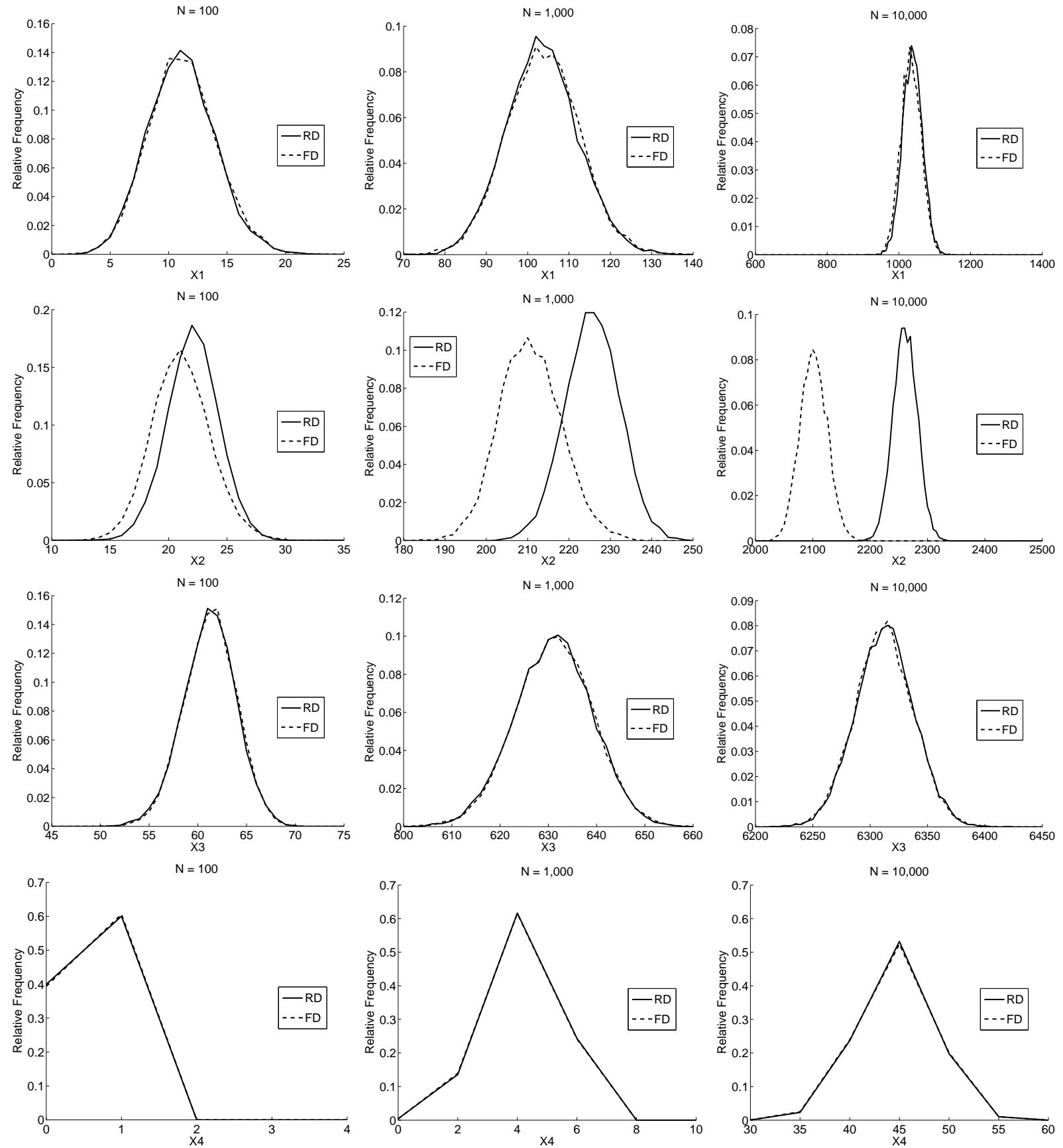

Figure 12: Histograms of the stochastic system with random delays (RD) and the stochastic system with fixed delays (FD) with $N=100$ (left column), $N=1,000$ (middle column), and $N=10,000$ (right column) at $t=25$. The random delay was chosen from a Gamma distribution with a mean of 5 and variance of 25.0. 

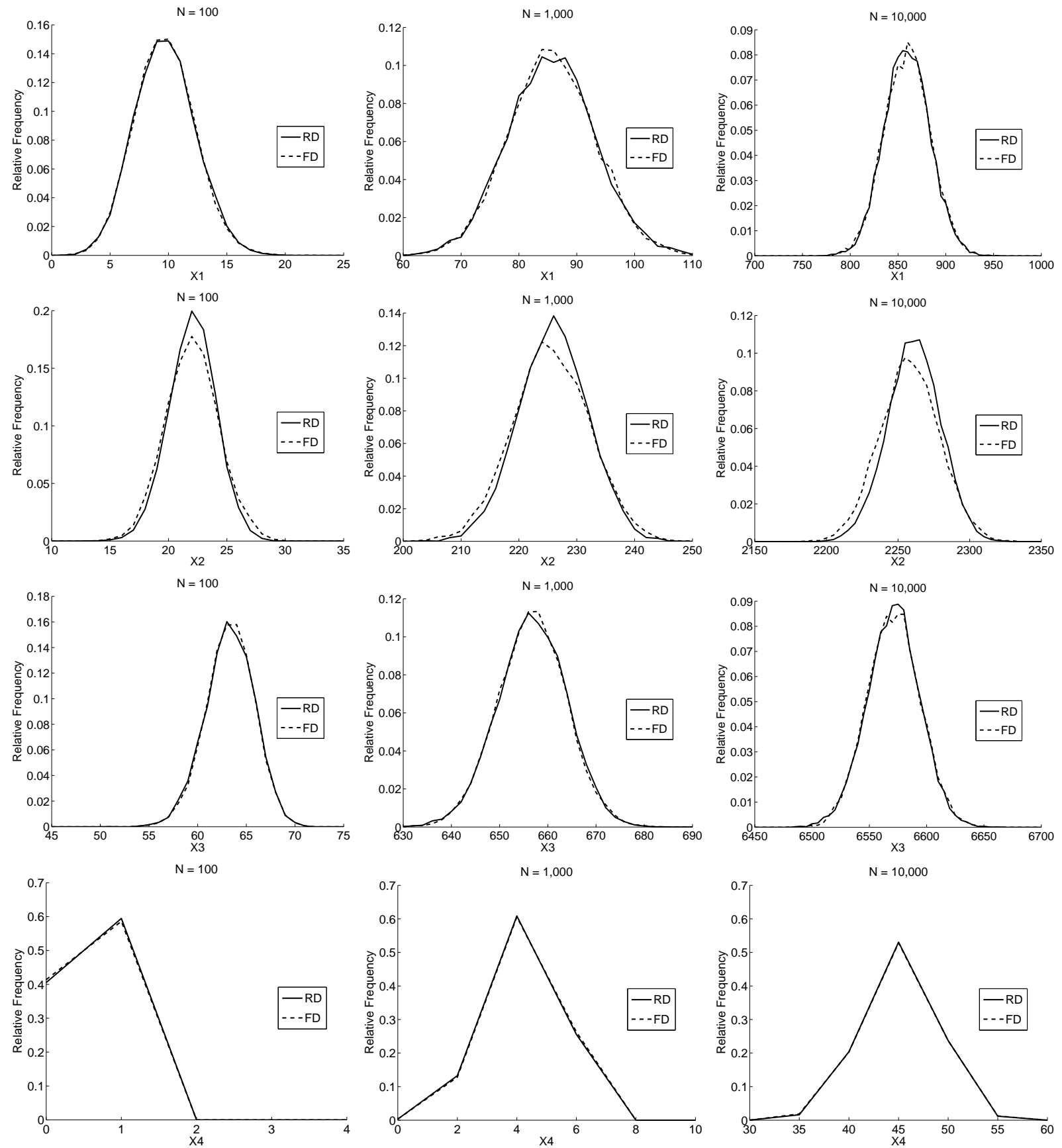

Figure 13: Histograms of the stochastic system with random delays (RD) and the stochastic system with fixed delays (FD) with $N=100$ (left column), $N=1,000$ (middle column), and $N=10,000$ (right column) at $t=50$. The random delay was chosen from a Gamma distribution with a mean of 5 and variance of 25.0. 


\subsection{The case with the variance of the random delay $\sigma^{2}=2.5$}

Next we considered the case where the probability density function $G(u ; \alpha, n)$ of the random delay is chosen such that $n=10$ and $\alpha=2$. The expected value of the delay remains at 5 , however the variance is now 2.5 , whereas previously the variance was 25.0. Figure 14 depicts the histograms for these two stochastic systems with $N=100$ (left column), $N=1,000$ (middle column), and $N=10,000$ (right column) at $t=25$. It is
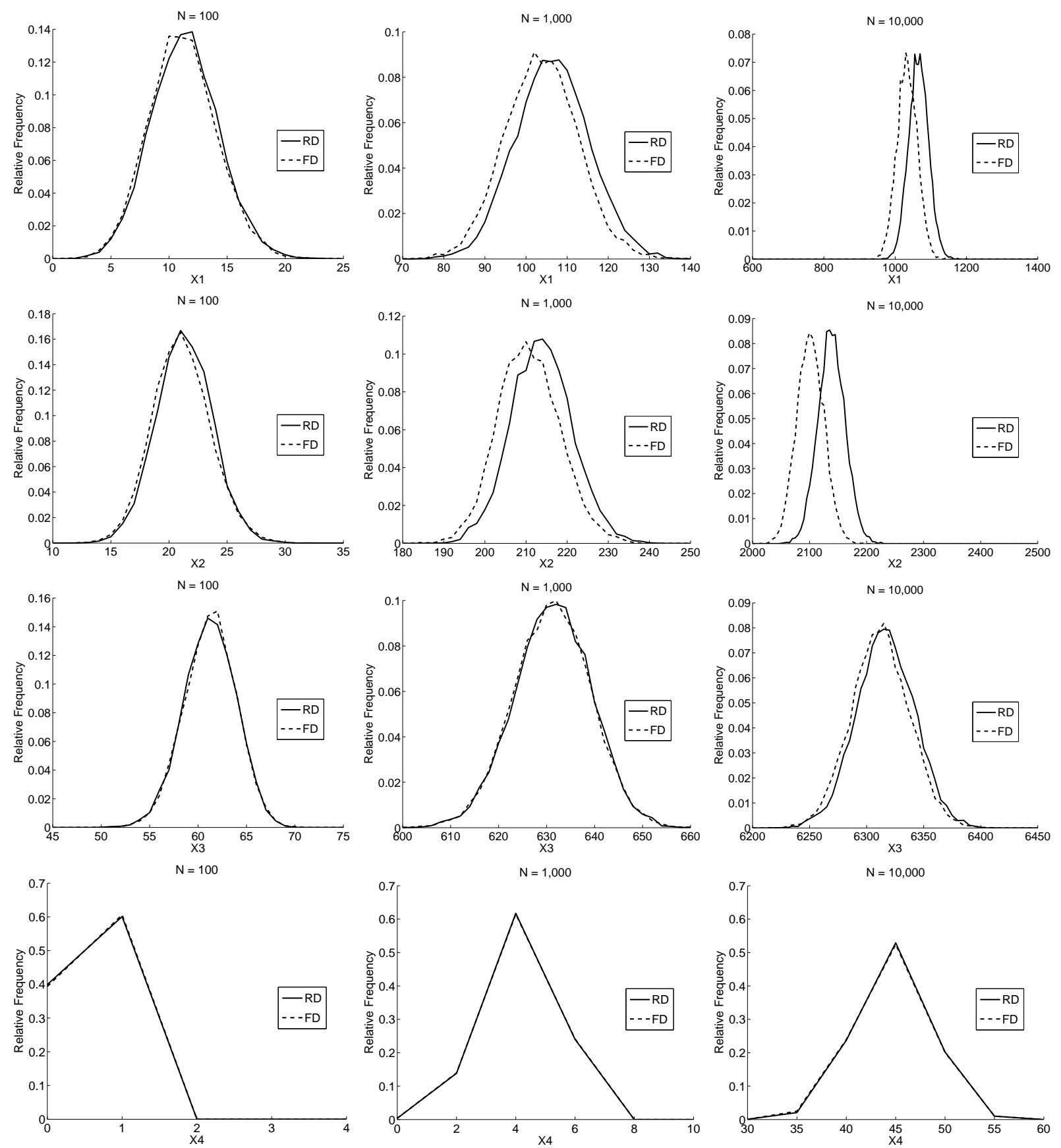

Figure 14: Histograms of the stochastic system with random delays (RD) and the stochastic system with fixed delays (FD) with $N=100$ (left column), $N=1,000$ (middle column), and $N=10,000$ (right column) at $t=25$. The random delay was chosen from a Gamma distribution with a mean of 5 and variance of 2.5.

seen from Figure 14 that the histograms for these two stochastic systems agree reasonably well for $X_{i}(t)$, $i=1,2,3,4$ at $t=25$ with sample size $N=100$. However, the histograms for $X_{1}(t), X_{2}(t)$ and $X_{3}(t)$ at 
$t=25$ deviate more as sample size increases, which is obvious for node 1 and more obvious for node 2 . Specifically, the histograms of these three nodes obtained for the stochastic system with a fixed delay have similar unimodal shape and dispersion as the corresponding ones obtained for the stochastic system with a random delay, but their mode values become smaller (i.e., shifted more to the left side as compared to the ones obtained for the stochastic system with a random delay) as the sample size increases. Figure 15 depicts the histograms for these two stochastic systems with $N=100$ (left column), $N=1,000$ (middle column), and $N=10,000$ (right column) at $t=50$. We observe from this figure that the histograms for
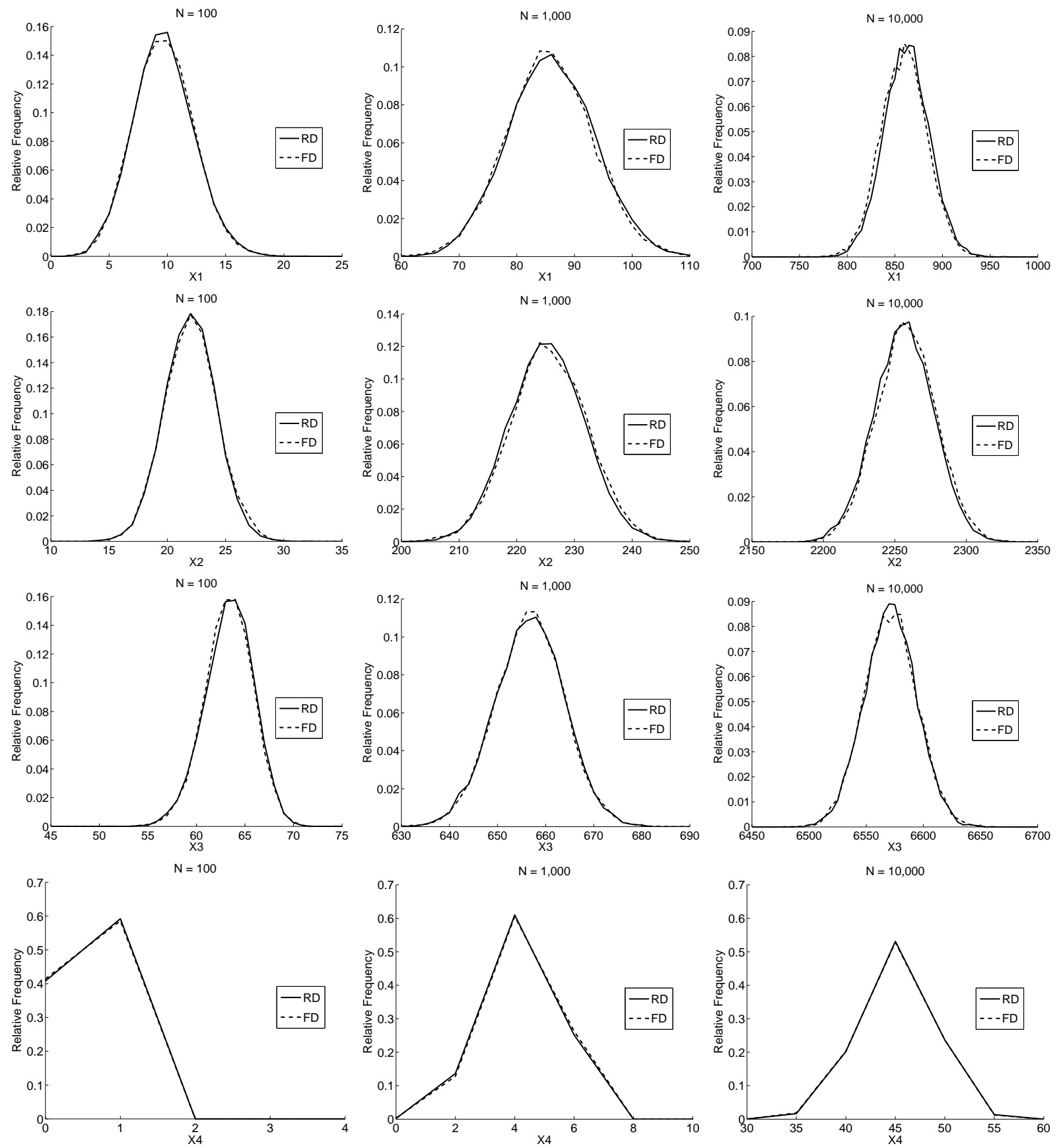

Figure 15: Histograms of the stochastic system with random delays (RD) and the stochastic system with fixed delays (FD) with $N=100$ (left column), $N=1,000$ (middle column), and $N=10,000$ (right column) at $t=50$. The random delay was chosen from a Gamma distribution with a mean of 5 and variance of 2.5 . 
these two stochastic systems agree well for all the four nodes at $t=50$ for all the sample sizes investigated. Overall, these figures indicate that the histograms for these two stochastic systems agree more compared to the corresponding ones in the previous section (i.e., the case with variance being 25). This is as expected, since as the variance decreases the random delay that is drawn during simulation is forced to be increasingly closer to its mean value. Hence, it is not surprising that the solutions are more similar when there is less variance.

\subsection{The case with the variance of the random delay $\sigma^{2}=0.25$}

We repeat the previous simulations, this time choosing the random delay from a Gamma distribution with mean 5 and variance 0.25 (i.e., the random delay has a probability density function $G(u ; 20,100)$ ). The histograms obtained for these two stochastic systems are depicted in Figures 16 and 17. From these it is seen that the histograms of all the nodes for these two stochastic systems agree well in all cases. This is as expected, since as the variance is sufficiently small (as for our case here) the random delay that is drawn during simulation is close to its mean value. In other words, the random delay is acting like a fixed delay and so it is not surprising that the histograms for these two stochastic systems are similar when the variance is sufficiently small. 

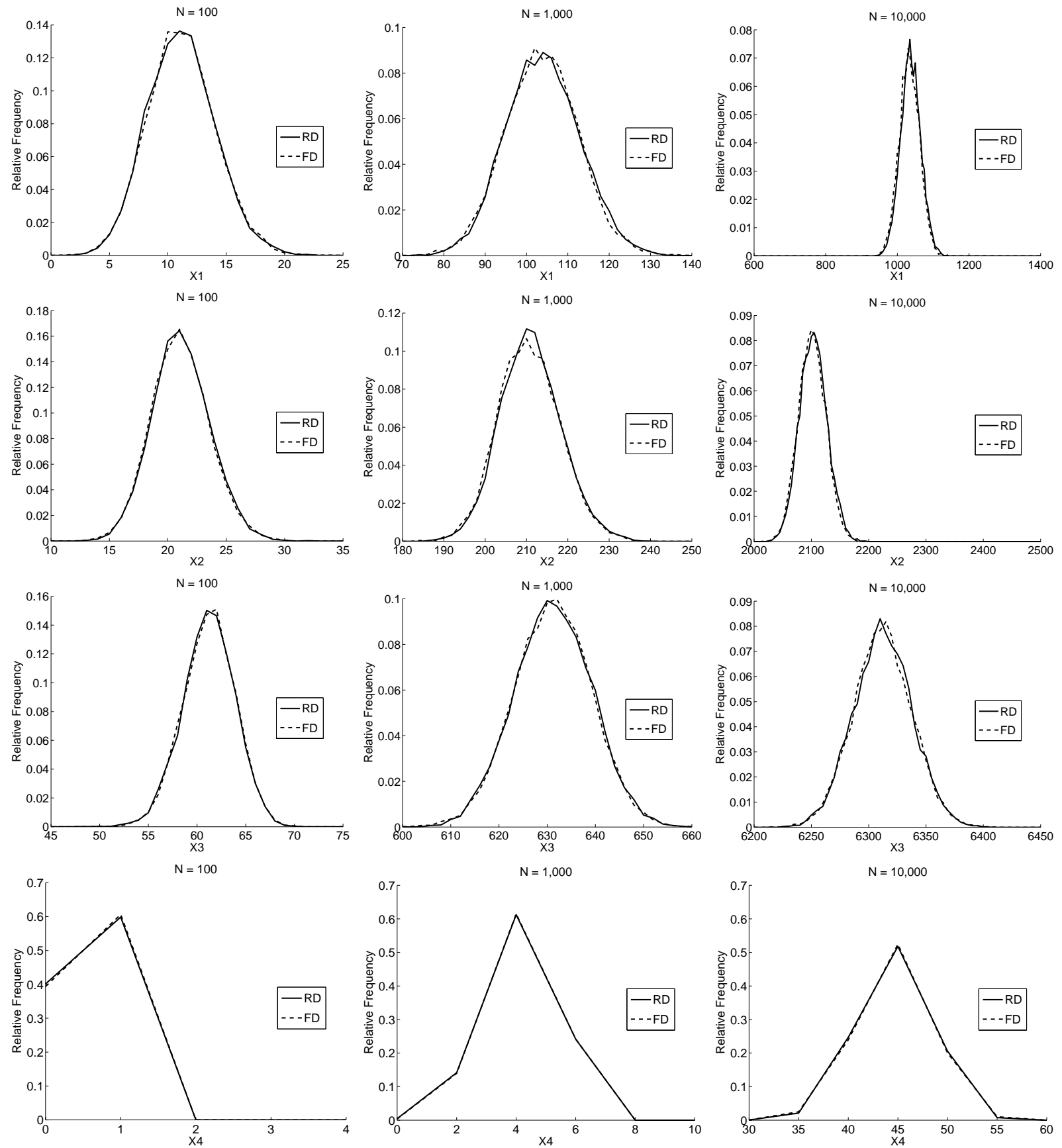

Figure 16: Histograms of the stochastic system with random delays (RD) and the stochastic system with fixed delays (FD) with $N=100$ (left column), $N=1,000$ (middle column), and $N=10,000$ (right column) at $t=25$. The random delay was chosen from a Gamma distribution with a mean of 5 and variance of 0.25 . 

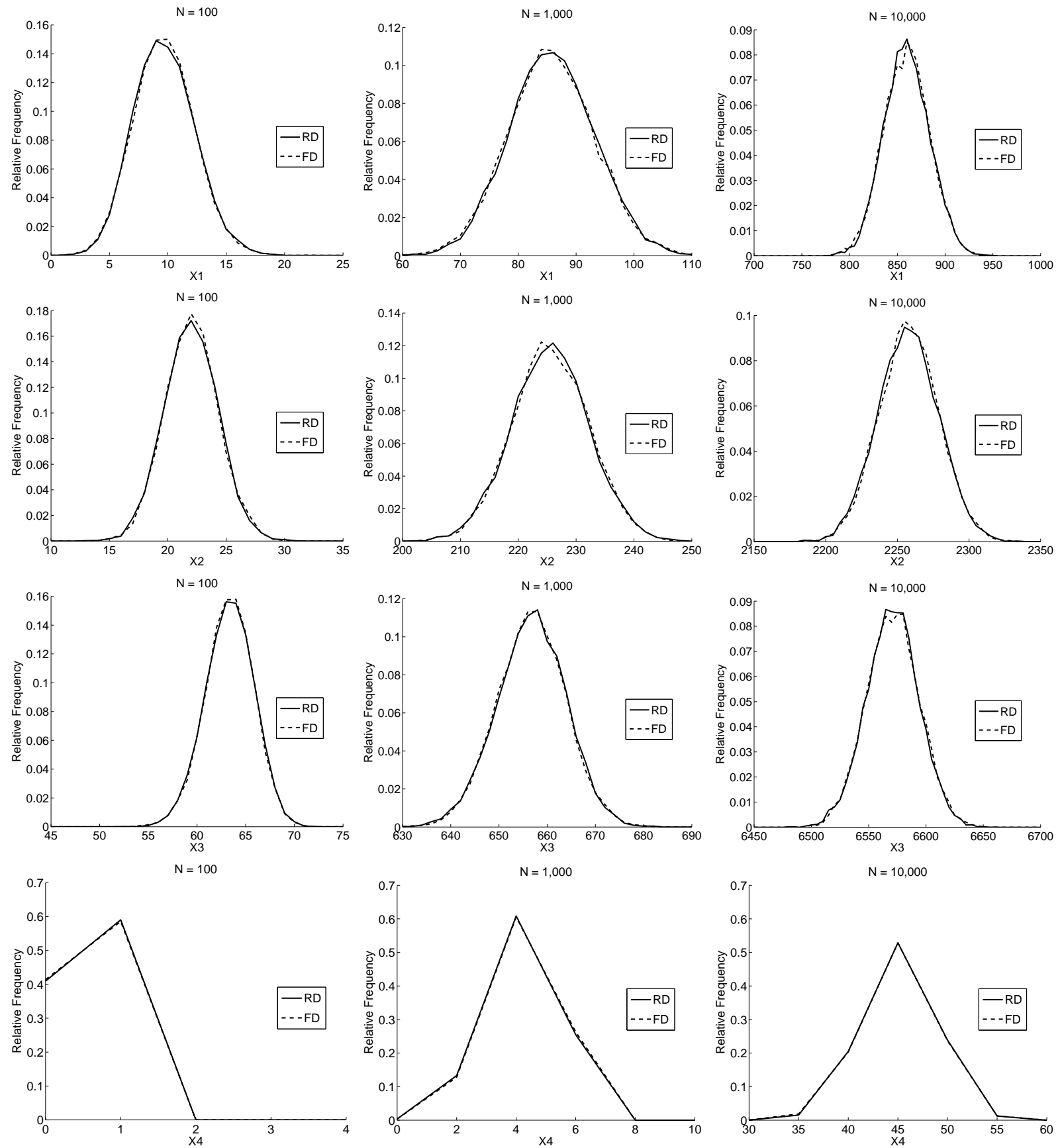

Figure 17: Histograms of the stochastic system with random delays (RD) and the stochastic system with fixed delays (FD) with $N=100$ (left column), $N=1,000$ (middle column), and $N=10,000$ (right column) at $t=50$. The random delay was chosen from a Gamma distribution with a mean of 5 and variance of 0.25 . 


\section{Conclusions}

In this paper we extended the stochastic pork production model in [4] to incorporate delays to account for the phenomenon that movement from one node to the next is often not instantaneous in practice due to the physical distance and/or some unexpected disruptions/interruptions. We considered two different types of delays, a fixed delay and a random delay, and numerically explored the corresponding deterministic approximations for these two resulting stochastic models. Numerical results reveal that when the sample size is sufficiently large the stochastic model with a fixed delay can be well approximated by a system of deterministic differential equations with a fixed delay. This conforms with the recent theoretical results presented in [9]. We also numerically showed that the mean solution of the stochastic model with a Gamma distributed random delay can be well approximated by the solution of a system of deterministic differential equations with a Gamma distributed delay when the sample size is sufficiently large. Hence, the system of deterministic differential equations with a Gamma distributed delay can be used as a possible corresponding deterministic one for this particular stochastic model with a Gamma distributed random delay (with the given parameter values and initial conditions).

In addition, we compared the stochastic model with a Gamma distributed random delay to the stochastic system constructed based on Kurtz's limit theorem from a system of deterministic differential equations with a Gamma distributed delay. Even though the same system of deterministic differential equations with a Gamma distributed delay can be used as the corresponding deterministic ones for these two stochastic systems, it was found that with the same sample size the histogram plots of the state solutions to the constructed stochastic system are more dispersed than the corresponding ones obtained for the stochastic model with a random delay. However, there is more agreement between the histograms of these two stochastic systems as the variance of the random delay decreases. We also found that with the same variance for the random delay the histogram plots for the stochastic model with a random delay are symmetric for all the sample sizes investigated, while those for the constructed stochastic system are asymmetric when the sample size is small, but become more symmetric as the sample size increases.

Finally we compared the histogram plots of the state solutions to the stochastic model with a fixed delay to those obtained for the stochastic model with a random delay, where the value of the fixed delay is chosen as the mean value of the random delay. Numerical results reveal that for those states most affected by the delay the histogram plots obtained for the stochastic system with a fixed delay have similar unimodal shapes and dispersion as the corresponding ones for the stochastic system with a random delay, but their mode values become smaller (i.e., shifted more to the left side as compared to the corresponding ones obtained for the stochastic system with a random delay) as the sample size increases. We also found that when the variance of the random delay is sufficiently small, the histograms of state solutions to the stochastic model with a fixed delay agree well with the corresponding ones obtained for the stochastic model with a random delay regardless of the sample size.

\section{Acknowledgements}

This research was supported in part by Grant Number NIAID R01AI071915-10 from the National Institute of Allergy and Infectious Diseases, in part by the Air Force Office of Scientific Research under grant number AFOSR FA9550-12-1-0188, and in part by the National Science Foundation under Research Training Grant (RTG) DMS-0636590.

\section{References}

[1] L.J.S. Allen, An Introduction to Stochastic Processes with Applications to Biology, Chapman \& Hall/CRC, Boca Raton, FL, 2011.

[2] D. Anderson, A modified next reaction method for simulating chemical systems with time dependent propensities and delays, The Journal of Chemical Physics, 127 (2007), 214107-1-214107-10.

[3] H. Andersson and T. Britton, Stochastic Epidemic Models and Their Statistical Analysis, SpringerVerlag, New York, 2000. 
[4] P. Bai, H.T. Banks, S. Dediu, A.Y. Govan, M. Last, A.L. Lloyd, H.K. Nguyen, M.S. Olufsen, G. Rempala and B.D. Slenning, Stochastic and deterministic models for agricultural production networks, Mathematical Biosciences and Engineering, 4 (2007), 373-402.

[5] H.T. Banks, A Functional Analysis Framework for Modeling, Estimation and Control in Science and Engineering, CRC Press/Taylor and Frances Publishing, 2012.

[6] H.T. Banks and F. Kappel, Spline approximations for functional differential equations, Journal of Differential Equations, 34 (1979), 496-522.

[7] M. Barrio, K. Burrage, A. Leier and T. Tian, Oscillatory regulation of Hes1: discrete stochastic delay modeling and simulation, PLoS Computational Biology, 2 (2006), 1017-1030.

[8] S.N. Busenberg and C.C. Travis, On the use of reducible functional differential equations in biological models, Journal of Mathematical Analysis and Applications, 89 (1982), 46-66.

[9] L. Bortolussi and J Hillston, Fluid approximations of CTMC with deterministic delays, Quantitative Evaluation of Systems (QEST), 2012 Ninth International Conference on, Sept 17-20, 2012, 53-62.

[10] D. Bratsun, D. Volfson, L.S. Tsimring and J. Hasty, Delayed-induced stochastic oscillations in gene regulation, PNAS, 102 (2005), 14593-14598.

[11] M. Gibson and J. Bruck, Efficient exact stochastic simulation of chemical systems with many species and many channels, The Journal of Physical Chemistry, 104 (2000), 1876-1899.

[12] S.N. Ethier and T.G. Kurtz, Markov Processes: Characterization and Convergence, J. Wiley \& Sons, New York, 1986.

[13] D.M. Fargue, Réducibilité des systèmes héréditaires a des systèmes dynamiques, C. R. Acad. Sci. Paris Ser. B, 277 (1973), 471-473.

[14] T.G. Kurtz, Solutions of ordinary differential equations as limits of pure jump Markov processes, $J$. Appl. Prob., 7 (1970), 49-58.

[15] T.G. Kurtz, Limit theorems for sequences of pure jump Markov processes approximating ordinary differential processes, J. Appl. Prob., 8 (1971), 344-356.

[16] T.G. Kurtz, Strong approximation theorems for density dependent Markov Chains, Stochastic Processes and their Applications, 6 (1978), 223-240.

[17] T.G. Kurtz, Approximation of Population Processes, SIAM, Pennsylvania, 1981.

[18] N. MacDonald, Time Lags in Biological Models, LN in Biomathematics Vol. 27, Springer-Verlag, Berlin, 1978.

[19] N. Minorsky, Self-excited oscillations in dynamical systems possessing retarded actions, Journal of Applied Mechanics, 9 (1942), A65-A71.

[20] N. Minorsky, On non-linear phenomenon of self-rolling, Proceedings of the National Academy of Sciences, 31 (1945), 346-349.

[21] N. Minorsky, Nonlinear Oscillations, Van Nostrand, New York, 1962.

[22] A.R. Ortiz, H.T. Banks, C. Castillo-Chavez, G. Chowell, and X. Wang, A deterministic methodology for estimation of parameters in dynamic Markov chain models, CRSC-TR10-07, N.C. State University, May, 2010; J. Biological Systems, 19 (2011), 71-100.

[23] J. Pahle, Biochemical simulations: stochastic, approximate stochastic and hybrid approaches, Brief Bioinform, 10 (2009), 53-64.

[24] M. Roussel and R. Zhu, Validation of an algorithm for delay stochastic simulation of transcription and translation in prokaryotic gene expression, Physical Biology, 3 (2006), 274-284. 
[25] S. Ruan, Delay differential equations in single species dynamics, in Delay Differential Equations and Applications, O. Arino et al. (eds.), Springer, Berlin, 2006, 477-517.

[26] R. Schlicht and G. Winkler, A delay stochastic process with applications in molecular biology, Journal of Mathematical Biology, 57 (2008), 613-648. 


\section{Appendix A.1: Comparison Of A Stochastic System With A Ran- dom Delay And A Constructed Stochastic System With Linear Transitions}

In this section we will partially repeat the analysis preformed in Section 3, however, instead of using the pork production model (with nonlinear transition rates), we will consider a simple stochastic system which has linear transitions. This new system has no known relevance to the pork production model. Rather, the goal of this analysis is to compare a stochastic system with linear transition rates with a random delay and a constructed stochastic system which has no delays in it. Following the procedure outlined in Section 3, we have that a stochastic system with a random delay can be approximated by a DDE with a distributed delay, where the delay kernel is the probability density function of the random delay. Making the assumption that the delay kernel is the probability density function of a Gamma distribution, we will again make use of the linear chain trick to transform the DDE system into an ODE system. At this point we will use the Kurtz limit theorem to construct a stochastic system which converges to the ODE system. Specifically, the purpose of this investigation is to compare the histograms that result from each of the stochastic systems in question.

Recall that in the case of the pork production model, the histograms from the two associated stochastic systems were shown to have considerable differences. We would like to gain insight as to whether this is primarily due to the nonlinearity of the transitions or if there may be a dependence of the types of approximations used for each of the stochastic systems. The motivation for this investigation is that in [26] it is stated that linear transitions are needed in order to obtain the approximation by a DDE with a distributed delay. Since the pork production model has nonlinear transitions, we would like to understand if the differences seen in the histograms might be due to this nonlinearity alone.

We begin by giving the DDE system

$$
\begin{aligned}
& \dot{c}_{1}(t)=-a c_{1}(t)+b \int_{-\infty}^{t} \alpha e^{-\alpha(t-s)} c_{1}(s) d s \\
& \dot{c}_{2}(t)=a c_{1}(t)-b \int_{-\infty}^{t} \alpha e^{-\alpha(t-s)} c_{1}(s) d s \\
& c_{1}(0)=c_{01}, \quad c_{2}(0)=c_{02} \\
& c_{1}(s)=0, \quad s<0
\end{aligned}
$$

which we will use as an example system in this section. Note that this is a linear system as desired, and that the delay kernel is of the form of $G(u ; \alpha, n)$ given in (14) where $n=1$. With the delay kernel in this form, we may use the linear chain trick as in Section 3.2 to obtain a system of ODE's.

$$
\begin{aligned}
\text { Letting } c_{3}(t)=b \int_{-\infty}^{t} \alpha e^{-\alpha(t-s)} c_{1}(s) d s, \text { we find } \\
\dot{c}_{1}(t)=-a c_{1}(t)+c_{3}(t) \\
\dot{c}_{2}(t)=a c_{1}(t)-c_{3}(t) \\
\dot{c}_{3}(t)=b \alpha c_{1}(t)-\alpha c_{3}(t) \\
c_{1}(0)=c_{01}, \quad c_{2}(0)=c_{02}, \quad c_{3}(0)=b \int_{-\infty}^{0} \alpha e^{\alpha s} c_{1}(s) d s .
\end{aligned}
$$

Let $\left(X_{1}, X_{2}, X_{3}\right)^{T}$ satisfy the stochastic system

$$
\begin{aligned}
& X_{1}(t)=X_{1}(0)-Y_{1}\left(\int_{0}^{t} a X_{1}(s) d s\right)+Y_{2}\left(\int_{0}^{t} X_{3}(s) d s\right) \\
& X_{2}(t)=X_{2}(0)+Y_{1}\left(\int_{0}^{t} a X_{1}(s) d s\right)-Y_{2}\left(\int_{0}^{t} X_{3}(s) d s\right) \\
& X_{3}(t)=X_{3}(0)+Y_{3}\left(\int_{0}^{t} b \alpha X_{1}(s) d s\right)-Y_{4}\left(\int_{0}^{t} \alpha X_{3}(s) d s\right),
\end{aligned}
$$

where $Y_{i}, i=1,2,3,4$ are independent unit Poisson processes and $X_{i}(0)=N c_{i}(0)$ for $i=1,2,3$. By using the Kurtz limit theorem we have that $\left(X_{1} / N, X_{2} / N, X_{3} / N\right)^{T}$ converges to the solution of (18) as $N \rightarrow \infty$. 
The final system yet to be mentioned is the stochastic system with a random delay. We will not attempt to write it out explicitly, but the system is essentially the same as (19), with the exception that every time transition 2 fires, the effect is delayed by a random amount of time. The value of the random time is sampled at every iteration, and is chosen from a Gamma distribution with probability density function $G(u ; \alpha, 1)$. This gives a mean value of $1 / \alpha$ and variance of $1 / \alpha^{2}$. For a more detailed explanation of a stochastic system with random delay we refer the reader back to Section 3.1.

For all the simulations below, the parameter values and initial conditions are given as follows.

$$
a=0.15, \quad b=0.2, \quad \alpha=0.4, \quad c_{01}=0.4, \quad c_{02}=0.6 .
$$

\section{Appendix A.2 Illustrations Of The System Dynamics}

To illustrate the dynamics of the system, Figure 18 depicts the solution to the ODE system (18) and 5 trials of the constructed stochastic system (19) with a sample size of $N=100$ and $N=1,000$. The ODE solution and the mean solution of 10,000 trials are given in Figure 19 for sample sizes of $N=100$ and $N=1,000$. As expected, as $N$ increases the trajectories of the stochastic simulations follow more closely the path of the solution to the ODE system. Additionally, the ODE solution provides a better approximation to the mean solution of the stochastic trials for larger $N$.

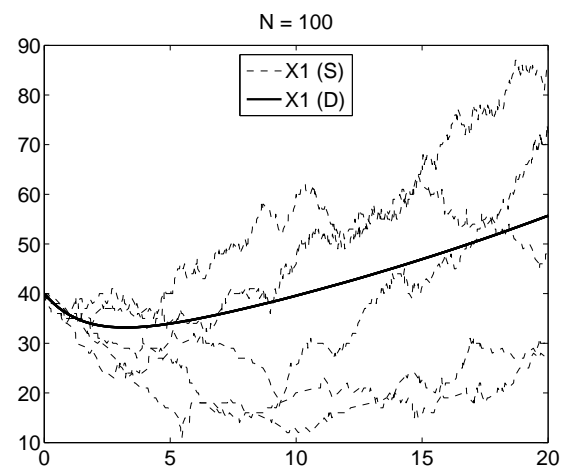

(a)

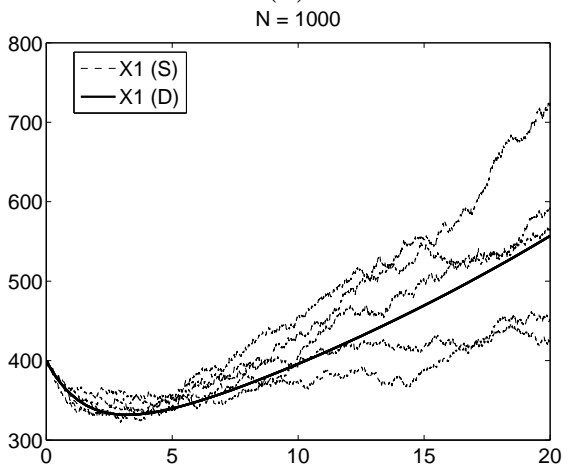

(c)

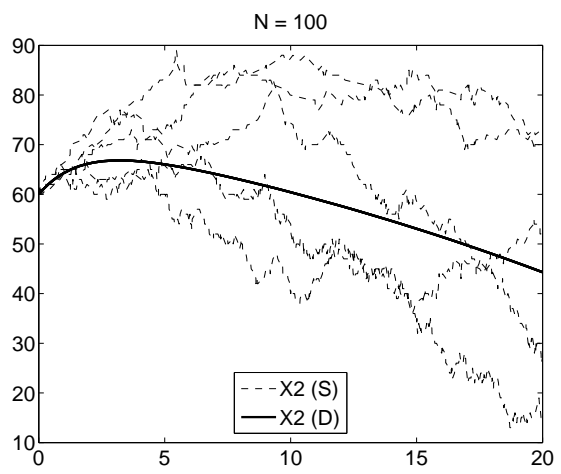

(b)

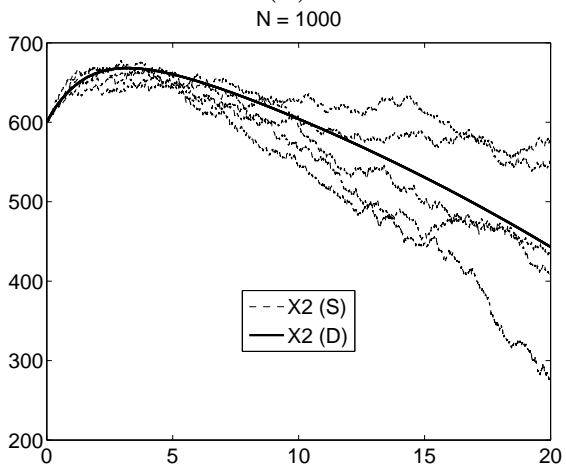

(d)

Figure 18: The ODE solution (D) and 5 trials of the constructed stochastic system (S) with a sample size of $N=100$ for (a) $X_{1}$ and (b) $X_{2}$, and a sample size of $N=1,000$ for (c) $X_{1}$ and (d) $X_{2}$. 


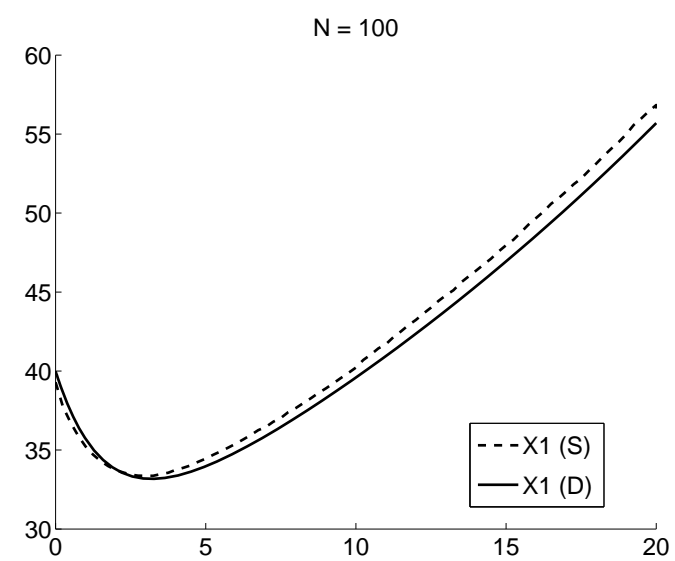

(a)

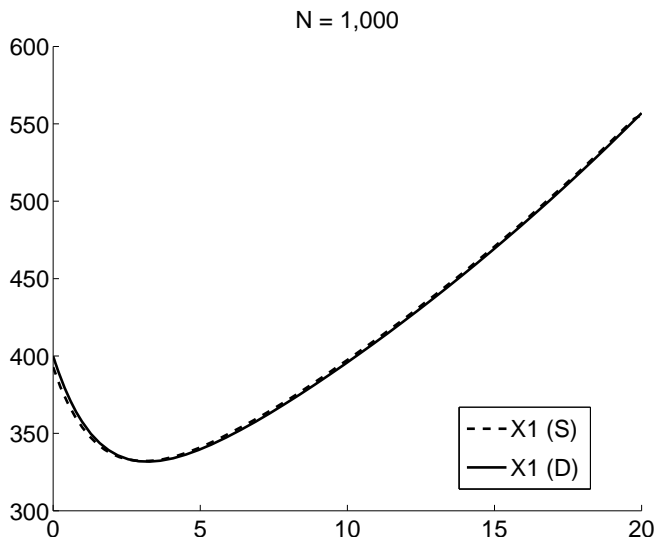

(c)

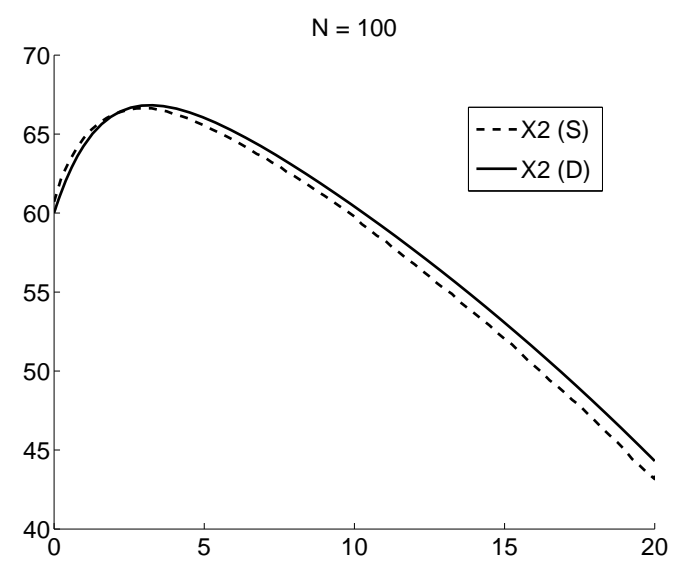

(b)

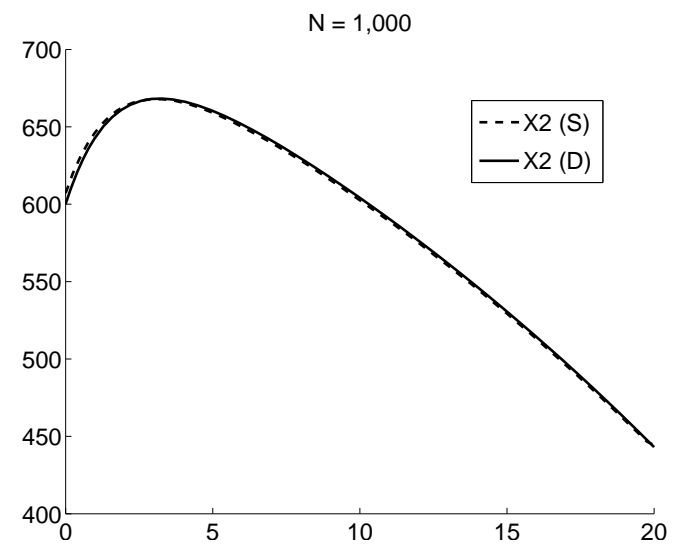

(d)

Figure 19: The mean solution of 10,000 trials for the constructed stochastic system (S) with a sample size of $N=100$ for (a) $X_{1}$ and (b) $X_{2}$ and with a sample size of $N=1,000$ for (c) $X_{1}$ and (d) $X_{2}$.

In Figure 20, the solution to the ODE system is shown along with 5 trials of the stochastic system with a random delay, again with a sample size of with a sample size of $N=100$ and $N=1,000$. The mean solution of 10,000 trials for this system is compared to the ODE solution and is given in Figure 21 for sample sizes of $N=100$ and $N=1,000$. The mean solution for a sample size of $N=10,000$ is considered as well, and is nearly identical to the case for $N=1,000$. As we observed with the constructed stochastic system, the ODE solution serves as a good approximation to the mean solutions, with the approximation improved for larger $N$. However, we note that the approximation is still very good for $N=100$. In [26] no explicit mention of the dependence on $N$ is made for the approximation to a stochastic system with random delays. Intuition would suggest that large sample sizes would be needed in order to approximate a discrete sampling of delays with a continuous delay kernel. To investigate this further we see in Figure 22 that the mean solution of the stochastic trials is not well approximated by the ODE solution as $t$ increases, when $N=30$. This suggest that the approximation does indeed, at least implicitly, have a dependence on the sample size. 


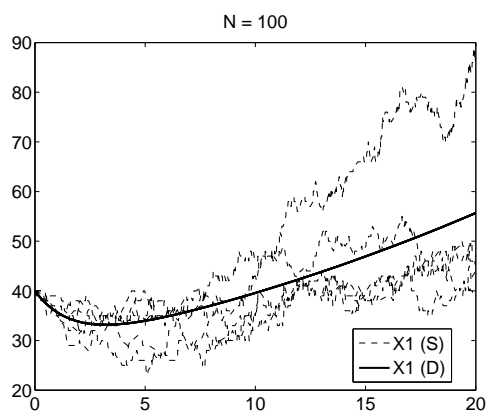

(a)

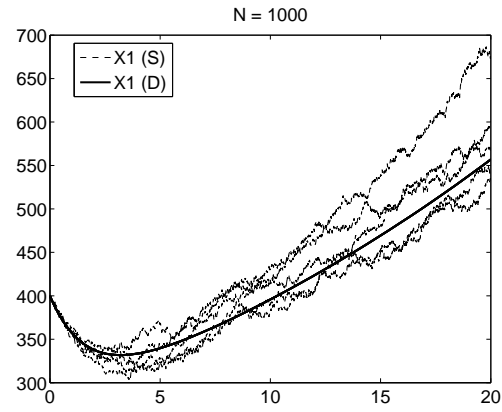

(c)

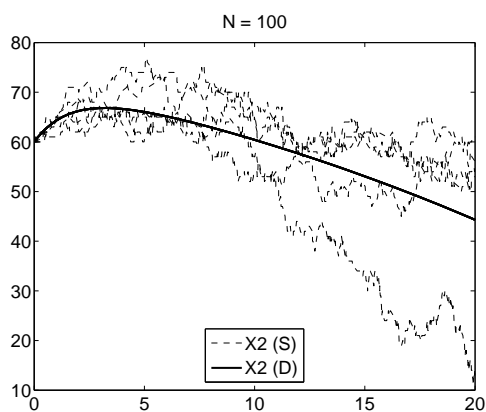

(b)

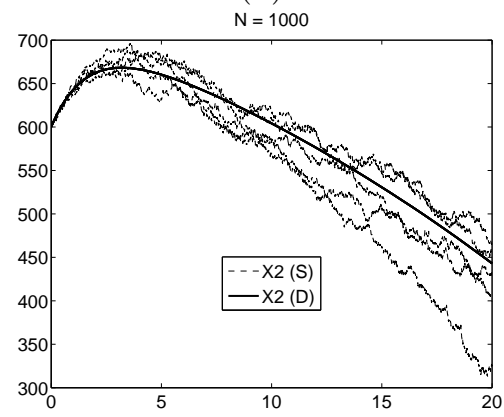

(d)

Figure 20: The ODE solution $(D)$ and 5 trials of the stochastic system with a random delay $(S)$ with a sample size of $N=100$ for (a) $X_{1}$ and (b) $X_{2}$, and a sample size of $N=1,000$ for (c) $X_{1}$ and (d) $X_{2}$.

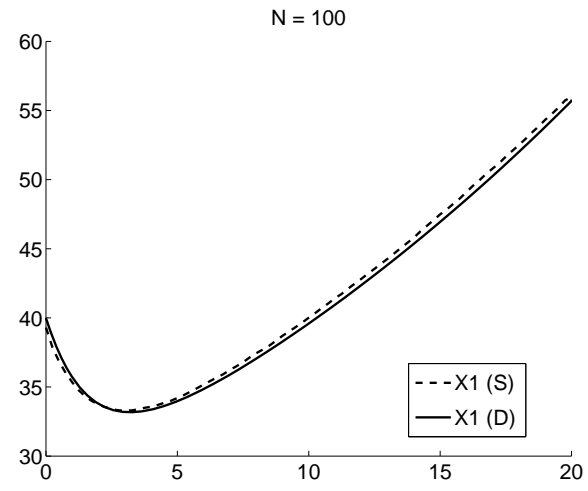

(a)

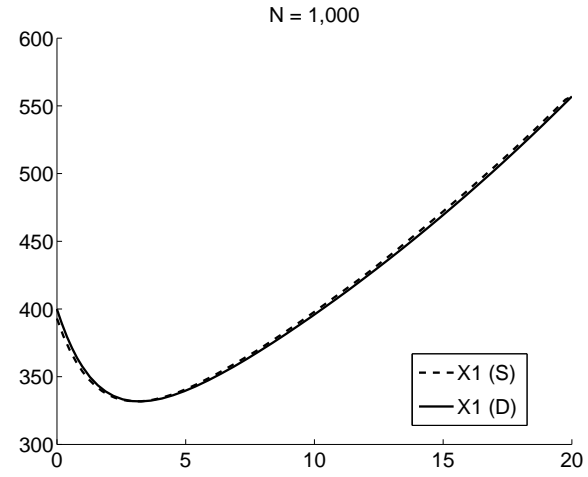

(c)

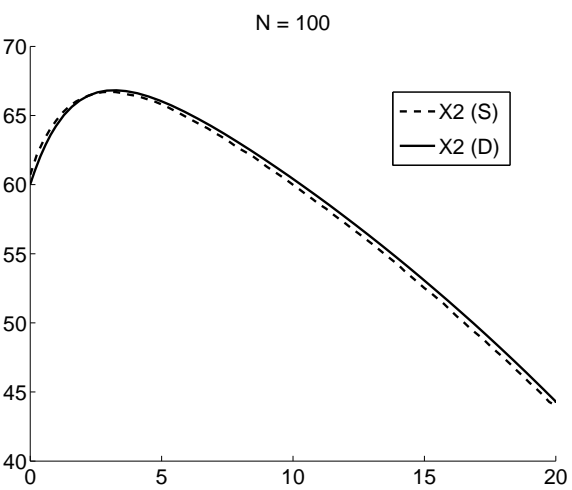

(b)

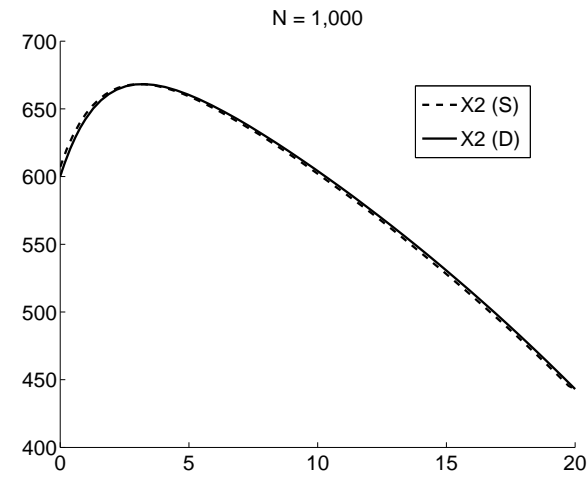

(d)

Figure 21: The mean solution of 10,000 trials for the stochastic system with a random delay (S) with a sample size of $N=100$ for (a) $X_{1}$ and (b) $X_{2}$ and with a sample size of $N=1,000$ for (c) $X_{1}$ and (d) $X_{2}$. 


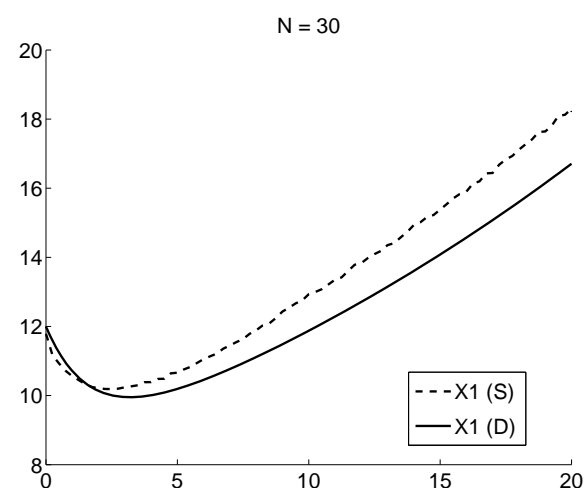

(a)

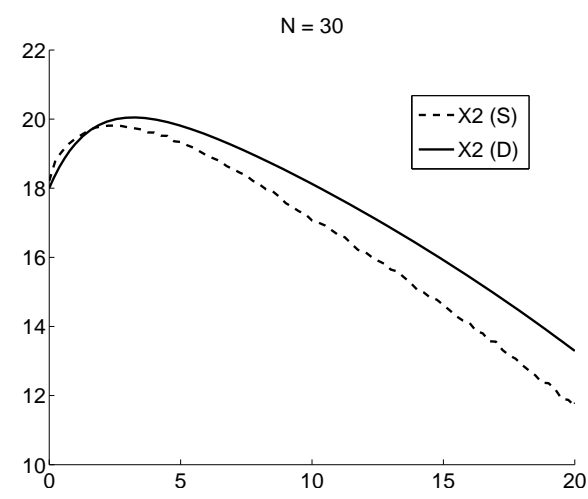

(b)

Figure 22: The mean solution of 10,000 trials for the stochastic system with a random delay (S) with a sample size of $N=30$ for (a) $X_{1}$ and (b) $X_{2}$.

\section{Appendix A.3 Comparison Of The Stochastic System With A Ran- dom Delay And The Constructed Stochastic System}

In this section we compare the histograms of the two stochastic systems. The histograms were calculated using 10,000 trials at times of $t=5,10,15$ and 20 for sample sizes of $N=100$ and 1,000. Figures 23, 24, and 25 show the histograms at a sample size of $N=100,1,000$, and 10,000 for the various times.

The figures of histograms seem to suggest that during the early stages of simulation $(t<10)$ the histograms are reasonably close. However, the histograms deviate increasingly as time increases. Looking back at Figures 18 and 20, we see that the trajectories deviate from the ODE solution as time increases. Presumable this is due to the delay in the system which has a mean value of 2.5 and variance of 6.25 . This may suggest that as the delay has more of an effect, the histograms diverge increasingly.

\section{Appendix A.4 Concluding Remarks}

These results show that when comparing a stochastic system with a random delay and a constructed stochastic system there are differences in the respective histograms, even in the case of linear transitions. This may suggest that the difference between the histograms is at least partially due to the different methods on which the stochastic systems are being approximated by deterministic systems. When making this same comparison in the pork production model with a mean delay of 5 and variance of 25 , a much higher level of disagreement between histograms was observed (see Figures 7 and 8). However, when the variance was taken as 2.5 (still with a mean of 5), the histograms of the Pork Production model display a level of agreement that is similar to what is shown here (see Figures 10 and 11). Moreover, when using different sample sizes for the two stochastic systems, the histograms of the pork production model again display agreement similar to the results given here (see Figure 9). Therefore, it may be plausible that the agreement between histograms is heavily dependent on the fact that two different methods were used to obtain a deterministic approximation (that being the method of Schlicht and Winkler, and the Kurtz limit theorem) as well as being dependent on both the variance of the delay and the sample sizes used, independent of whether linear or nonlinear transitions appear in the model. 


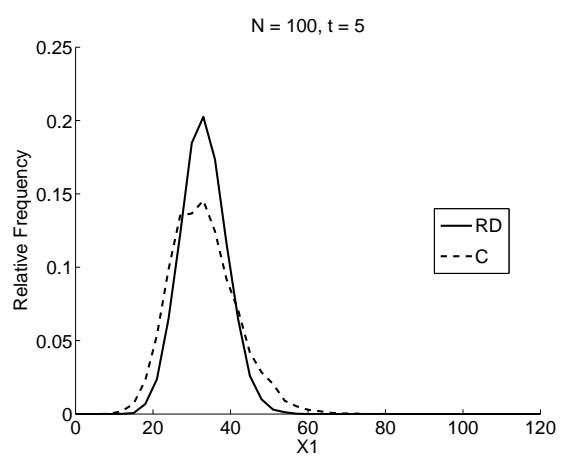

(a)

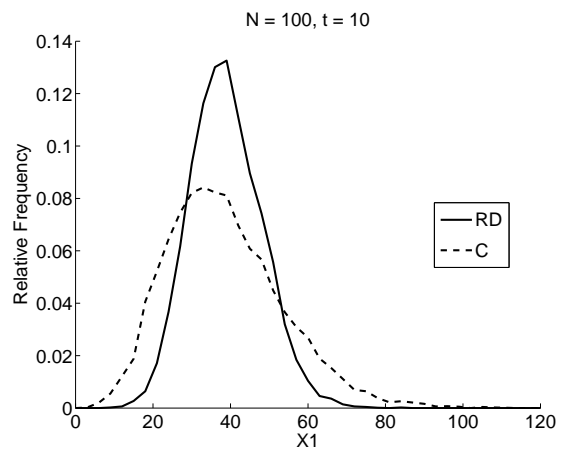

(c)

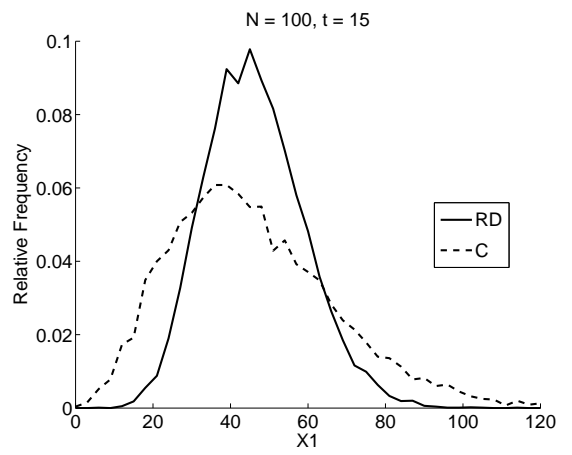

(e)

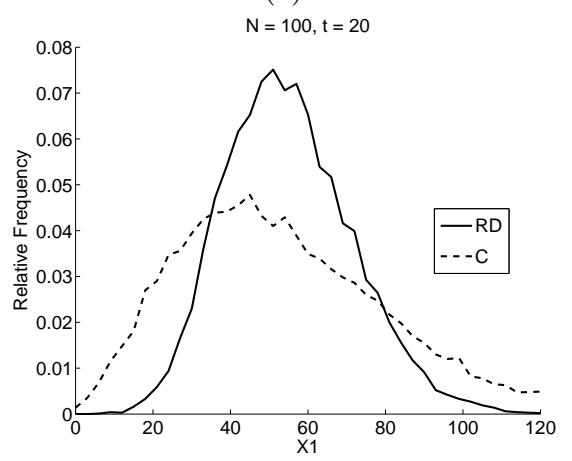

(g)

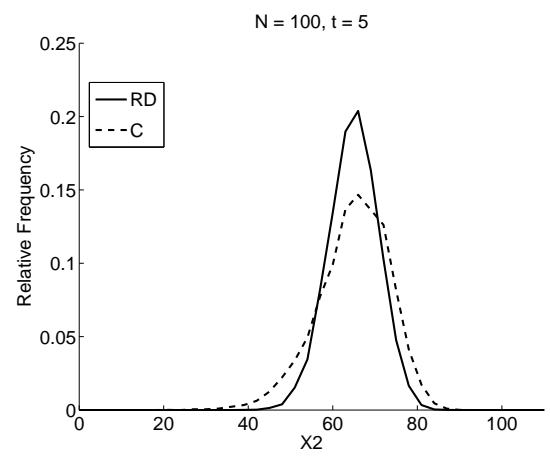

(b)

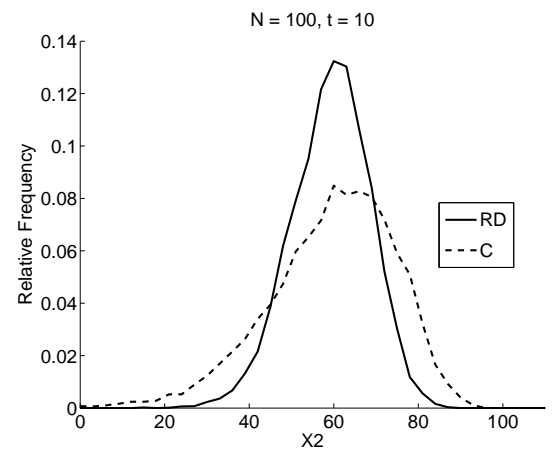

(d)

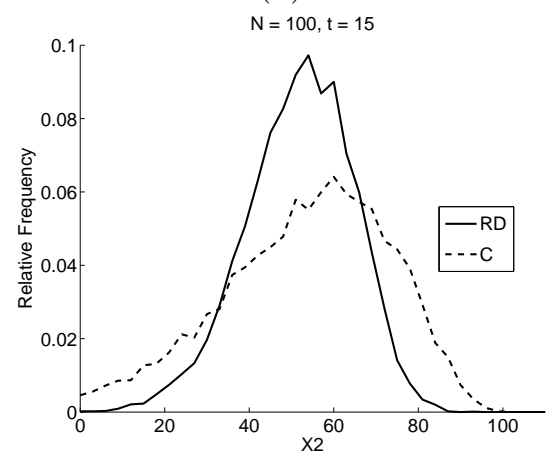

(f)

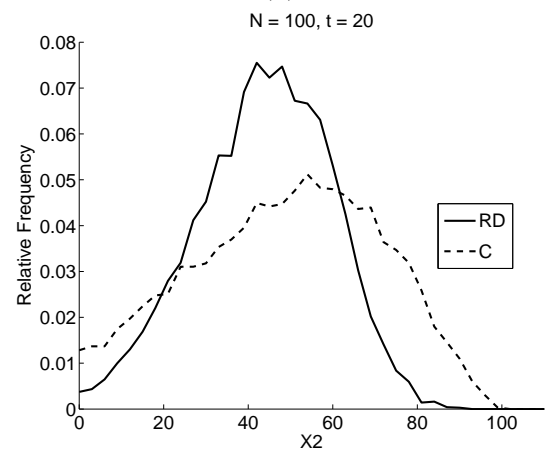

(h)

Figure 23: Histograms of the constructed stochastic system $(C)$ and the stochastic system with a random delay $(R D)$ for a sample size of $N=100$. 


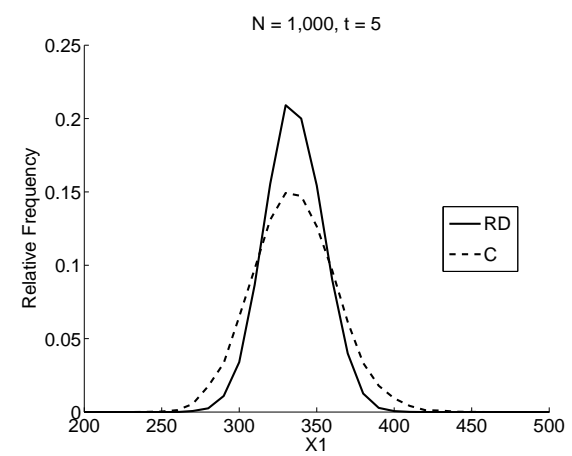

(a)

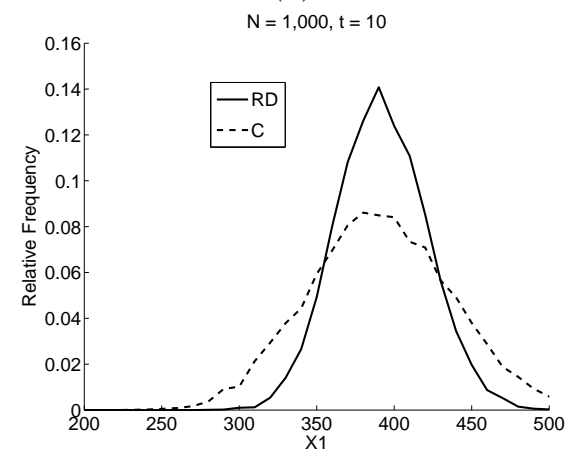

(c)

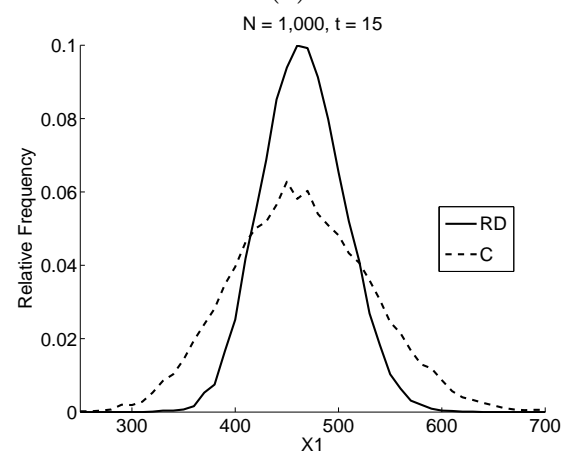

(e)

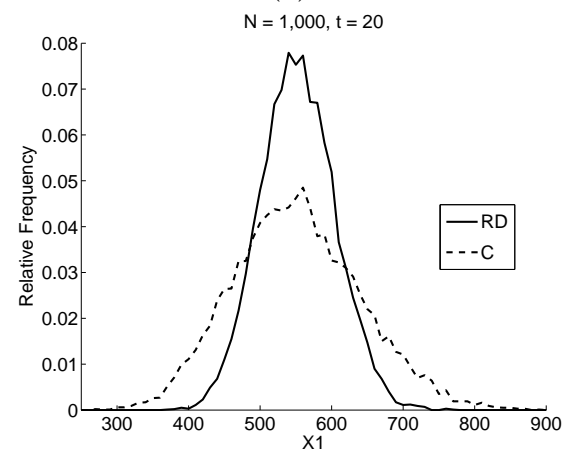

(g)

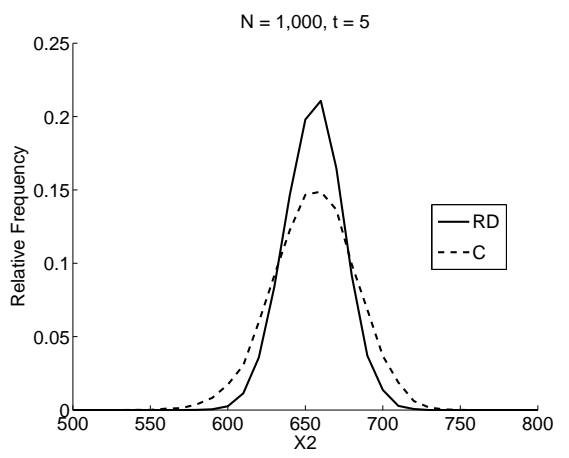

(b)

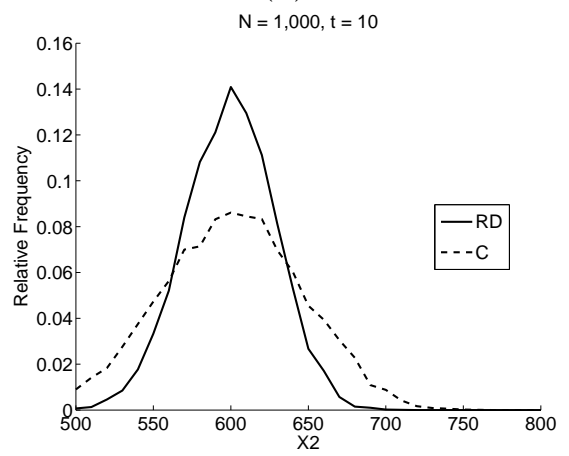

(d)

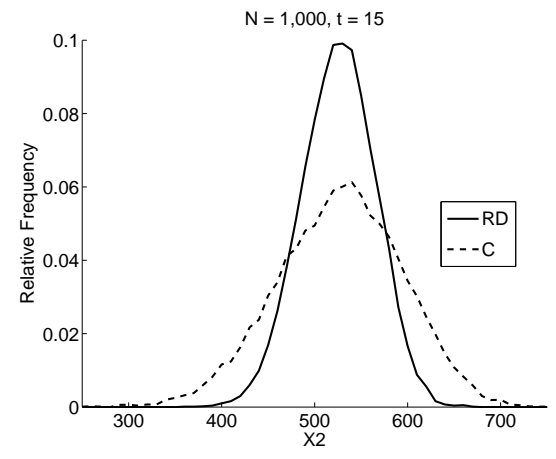

(f)

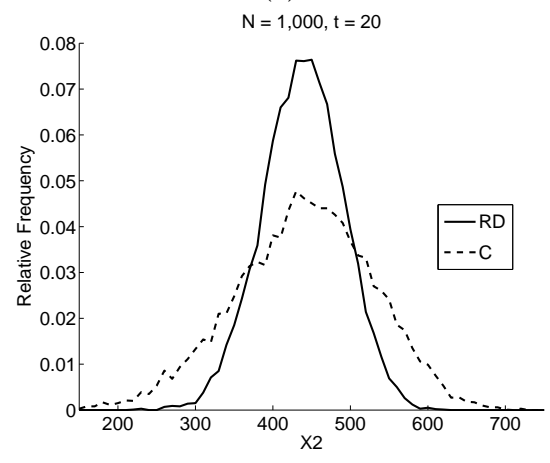

(h)

Figure 24: Histograms of the constructed stochastic system $(C)$ and the stochastic system with a random delay (RD) for a sample size of $N=1,000$. 


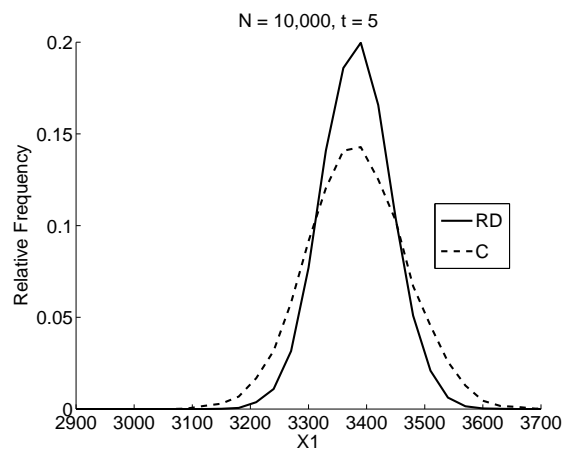

(a)

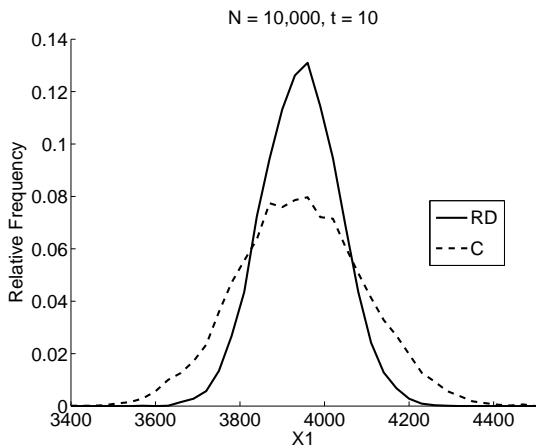

(c)

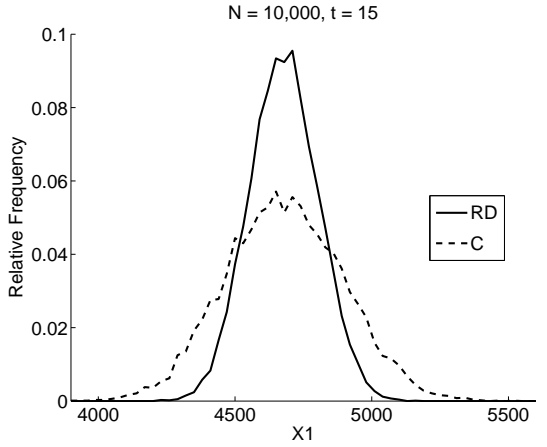

(e)

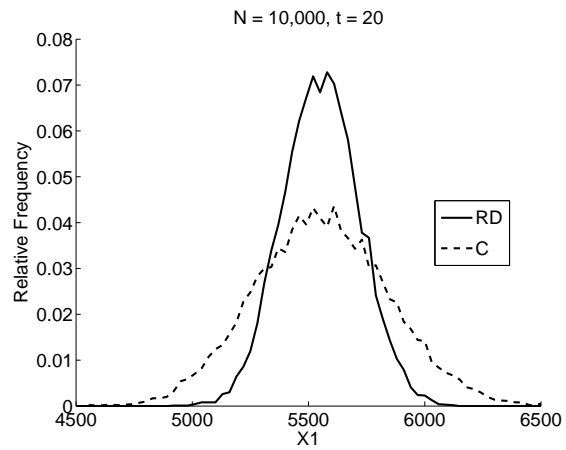

$(\mathrm{g})$

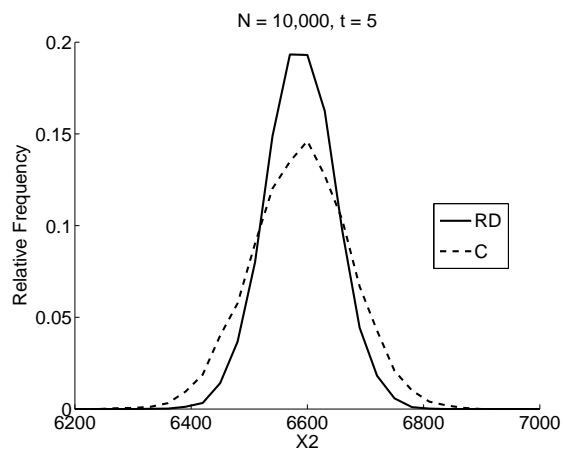

(b)

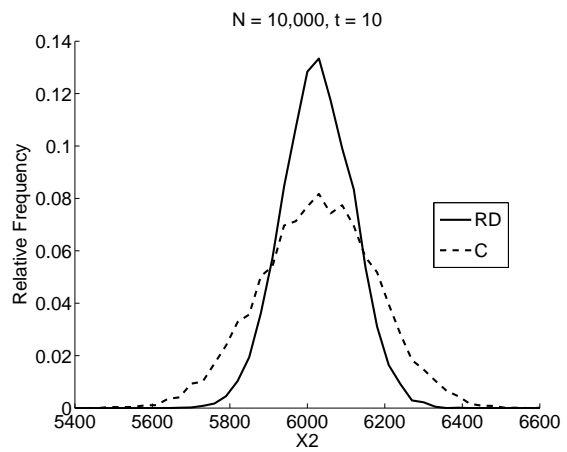

(d)

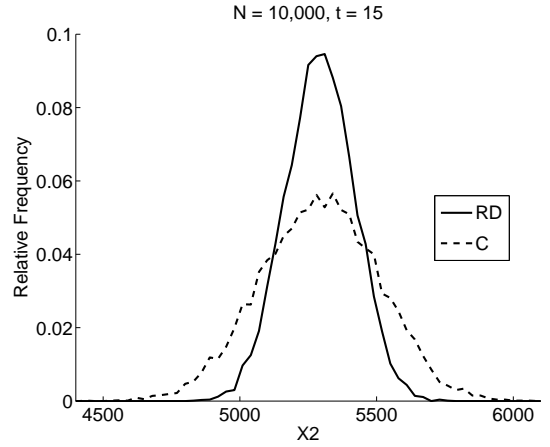

(f)

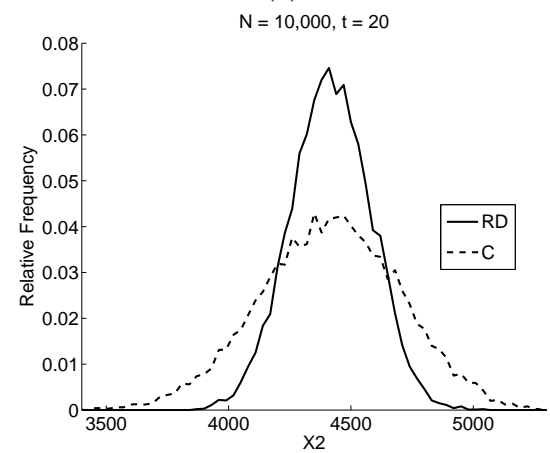

(h)

Figure 25: Histograms of the constructed stochastic system $(C)$ and the stochastic system with a random delay (RD) for a sample size of $N=10,000$. 